\title{
Statistical Assessment of Water Quality Parameters for Pollution Source Identification in Sukhnag Stream: An Inflow Stream of Lake Wular (Ramsar Site), Kashmir Himalaya
}

\author{
Salim Aijaz Bhat, ${ }^{1}$ Gowhar Meraj, ${ }^{2}$ Sayar Yaseen, ${ }^{1}$ and Ashok K. Pandit ${ }^{1}$ \\ ${ }^{1}$ Aquatic Ecology Laboratory, Centre of Research for Development (CORD), Department of Environmental Science, \\ University of Kashmir, Jammu and Kashmir 190006, India \\ ${ }^{2}$ GIS Laboratory, Department of Earth Sciences, University of Kashmir, Jammu and Kashmir 190006, India
}

Correspondence should be addressed to Salim Aijaz Bhat; salimaijaz@gmail.com

Received 1 June 2013; Accepted 4 December 2013; Published 20 January 2014

Academic Editor: Guangliang Liu

Copyright (C) 2014 Salim Aijaz Bhat et al. This is an open access article distributed under the Creative Commons Attribution License, which permits unrestricted use, distribution, and reproduction in any medium, provided the original work is properly cited.

\begin{abstract}
The precursors of deterioration of immaculate Kashmir Himalaya water bodies are apparent. This study statistically analyzes the deteriorating water quality of the Sukhnag stream, one of the major inflow stream of Lake Wular. Statistical techniques, such as principal component analysis (PCA), regression analysis, and cluster analysis, were applied to 26 water quality parameters. PCA identified a reduced number of mean 2 varifactors, indicating that $96 \%$ of temporal and spatial changes affect the water quality in this stream. First factor from factor analysis explained $66 \%$ of the total variance between velocity, total- $\mathrm{P}, \mathrm{NO}_{3}-\mathrm{N} \mathrm{Ca}^{2+}, \mathrm{Na}^{+}$, TS, TSS, and TDS. Bray-Curtis cluster analysis showed a similarity of $96 \%$ between sites IV and V and $94 \%$ between sites II and III. The dendrogram of seasonal similarity showed a maximum similarity of $97 \%$ between spring and autumn and $82 \%$ between winter and summer clusters. For nitrate, nitrite, and chloride, the trend in accumulation factor (AF) showed that the downstream concentrations were about 2.0,2.0, and 2.9, times respectively, greater than upstream concentrations.
\end{abstract}

\section{Introduction}

River water quality is of great environmental concern since it is one of the major available fresh water resources for human consumption $[1,2]$. Throughout the history of human civilization, rivers have always been heavily exposed to pollution, due to their easy accessibility to disposal of wastes. However, after the industrial revolution the carrying capacity of the rivers to process wastes reduced tremendously [3, 4]. Anthropogenic activities such as urban, industrial, and agricultural as well as natural processes, such as precipitation inputs, erosion, and weathering of crustal materials affect river water quality and determine its use for various purposes [1-5]. The usage also depends upon the linkages (channels) in the river system, as inland waterways play a major role in the assimilation and transportation of contaminants from a number of sources [6-8]. Besides linkages, the seasonal variation in precipitation, surface runoff, interflow, groundwater flow, and pumped in and out flows also have a strong effect on the concentration of pollutants in rivers [9-12]. In view of the limited stock of freshwater worldwide and the role that anthropogenic activities play in the deterioration of water quality, the protection of these water resources has been given topmost priority in the 21st century [13-15]. Research-wise, one of the important stages in the protection and conservation of these resources is the spatiotemporal analysis of water and sediment quality of the aquatic systems [16]. The nonlinear nature of environmental data makes spatio-temporal variations of water quality often difficult to interpret and for this reason statistical approaches are used for providing representative and reliable analysis of the water quality [17]. Multivariate statistical techniques such as cluster analysis (CA) and factor analysis (FA) have been widely used as unbiased methods in analysis of water quality data for drawing out meaningful conclusions $[18,19]$. Also it has been widely used to characterize and evaluate water quality for analyzing spatio-temporal variations caused by natural and anthropogenic processes [20-22]. In this paper we present 
a methodology for examining the impact of all the sources of pollution in Sukhnag stream (Kashmir Himalayas) and to identify the parameters responsible for spatiotemporal variability in water quality using CA and FA.

\section{Materials and Methods}

\subsection{Materials}

2.1.1. Study Area. The present study was carried out on Sukhnag stream in Kashmir Himalaya. It is among the five major inflows of the Lake Wular. This lake is the largest fresh water lake of Indian subcontinent and has been designated as a Ramsar site in 1990 under the Ramsar convention of 1975. The Sukhnag, a torrential stream, flows through Budgam district, in the state of Jammu and Kashmir (Figure 1). It flows from the mountain reaches of the Pir Panjal mountain range located in the southwest of Beerwah town. The Sukhnag stream drains the famous Toshmaidan region in the higher locales of Pir Panjal range. It has a glacial origin and covers a distance of about $51 \mathrm{kms}$ from head to mouth. Descending from the mountains, the Sukhnag passes through a sand choked bed across the Karewas, finally merging with the outlet of Hokersar wetland (Ramsar site). The Sukhnag drainage system spreads over an area of about $395.03 \mathrm{~km}^{2}$ and about 1551 streams cascade the waters for the whole watershed into this stream. During flash rains the water in this stream flows with the tremendous velocity in the upper reaches causing soil wastage of the left and right embankments of the stream and greatly damaging standing crops, plantation, houses, and road communication. The stream passes through a large area of high socioeconomic importance to North Kashmir. These areas include Rangzabal, Zagu, Bras, Arizal, Chill, Zanigam, Sail, Kangund, Goaripora, Siedpora, Beerwah, Aarwah, Aripanthan, Rathson, Makhama, Nawpora, Check-kawosa, Botacheck, and Narbal. The stream serves as a life line of this vast area as it serves as a source of water for both domestic and agricultural purposes. The current study is therefore a step forward in addressing the deteriorating conditions of the stream so as to recommend concrete measures for its sustainable management.

\subsection{Methods}

2.2.1. Sampling and Analysis. Samples were taken flow proportionately (i.e., more frequently during peak flow periods than during low flow periods) to capture nutrient pulses during runoff events from February 2011 to January 2012. The surface water samples were collected in midchannel points between 10.00 and 12.00 hours from each of the sampling sites and placed in prerinsed polyethylene and acid-washed bottles for the laboratory investigations. The parameters such as depth, transparency, temperature, $\mathrm{pH}$, and conductivity were determined on the spot while the rest of the parameters were determined in the laboratory. These include orthophosphorus, total phosphorus, ammoniacal nitrogen, nitrite-nitrogen, nitrate-nitrogen, organic nitrogen (Kjeldahl nitrogen minus ammonical nitrogen), alkalinity, free $\mathrm{CO}_{2}$, conductivity, chloride, total hardness, calcium hardness, magnesium hardness, sodium, and potassium. They were determined in the laboratory within 24 hours of sampling by adopting standard methods of Golterman and Clymo (1969) and APHA (1998) [23-25].

2.2.2. Statistical Analysis. Data for physicochemical parameters of water samples were presented as mean values and analyzed using descriptive analysis. We used coefficient of correlation (CV) and $t$-test, for describing the temporal variations of the observed water quality parameters. Prior to investigating the seasonal effect on water quality parameters, we divided the whole observation period into four fixed seasons: spring (March, April, and May), summer (June, July, and August), autumn (September, October, and November), and winter (December, January, and February). Regression analysis (RA) was carried out in order to know the nature and magnitude of the relationship among various physicochemical parameters. First, we determined the best-fit model (the largest $R^{2}$ ) for exploring whether there was any significant relationship among water quality parameters or not.

Accumulation factor (AF), the ratio of the average level of a given parameter downstream (following source discharge) to the corresponding average level upstream (prior to the source discharge) [26], was used to estimate the degree of contamination due to anthropogenic inputs.

The degree of river recovery capacity (RRC) for this stream was calculated using the mathematical equation by Ernestova and Semenova [27] and modified by Fakayode [26]; that is,

$$
\mathrm{RRC}=\frac{S_{0}-S_{1}}{S_{0}} \times 100(\text { expressed in } \%),
$$

where $S_{0}$ is the level of a parameter downstream (i.e., immediately after the discharge point) and $S_{1}$ is the corresponding average level upstream where the water is relatively unpolluted.

2.2.3. Multivariate Statistical Methods. With the objective of evaluating significant differences among the sites for all water quality variables, data was analyzed using one-way analysis of variance (ANOVA) at $0.05 \%$ level of significance [28]. Stream water quality was subjected to two multivariate techniques: cluster analysis (CA) and principal component analysis (PCA) [29]. CA and PCA explore groups and sets of variables with similar properties, thus potentially allowing us to simplify our description of observations by allowing us to find the structure or patterns in the presence of chaotic or confusing data [30]. All statistical analyses were performed using the SPSS (v. 16) and PAST (v. 1.93) software applications.

Cluster Analysis (CA). Cluster analysis is a multivariate statistical technique, which allows the assembling of objects based on their similarity. CA classifies objects, so that each object is similar to the others in the cluster with respect to a predetermined selection criterion. Bray-Curtis cluster analysis is the most common approach of $\mathrm{CA}$, which provides intuitive similarity relationships between any one sample and the entire dataset and is typically illustrated by a dendrogram (tree diagram). The dendrogram provides a visual summary 


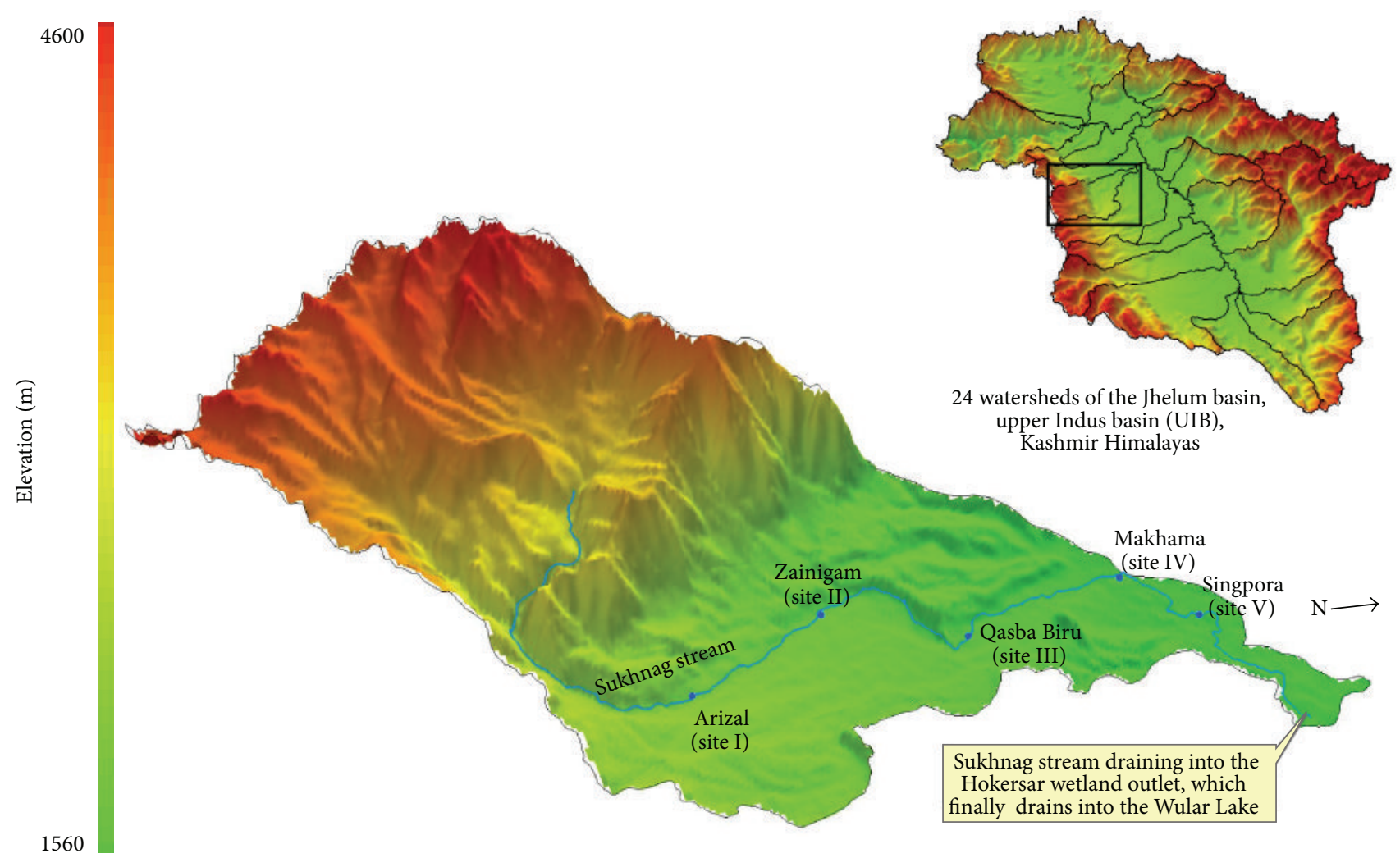

Sukhnag watershed

- Sampling sites

$\square$ Watershed boundary

FIGURE 1: Location of the study area with respect to Jhelum basin watersheds and surface water quality monitoring stations in the Sukhnag watershed.

of the clustering processes, presenting a picture of the groups and their proximity with a dramatic reduction in dimensionality of the original data [31].

Factor Analysis/Principal Component Analysis (PCA). Factor analysis is applied to reduce the dimensionality of a data set consisting of large number of interrelated variables, and this reduction is achieved by transforming the data set into a new set of variables-the principal components (PCs), which are orthogonal (noncorrelated) and are arranged in decreasing order of importance. In this study we used principal component analysis (PCA) of factor analysis. The PCA is a data reduction technique and suggests how many varieties are important to explain the observed variance in the data. Mathematically, PCs are computed from covariance or other cross-product matrixes, which describe the dispersion of the multiple measured parameters to obtain eigenvalues and eigenvectors. Moreover, these are the linear combinations of the original variables and the eigenvectors [32]. PCA can be used to reduce the number of variables and explain the same amount of variance with fewer variables (principal components) [33]. Also PCA attempts to explain the correlation between the observations in terms of the underlying factors, which are not directly observable [34].
Prior to modeling, all the nutrient concentrations were log-transformed to make the distribution closer to the normal. Statistical conclusions and tests were made on the basis of a multiparametric model. We have used CV, $t$ test, ANOVA, RA, CA, and PCA to evaluate the impact of anthropogenic activities and spatio-temporal variations on physicochemical characteristics of Sukhnag stream.

\section{Results and Discussion}

The mean values of physicochemical parameters at different sampling sites in Sukhnag stream during the period of 12 months (February 2011-January 2012) are presented in Table 1. Water temperature, $\mathrm{pH}$, and $\mathrm{DO}$ demonstrated a seasonal cycle during the period of study. High temperature values were recorded $\left(24.33 \pm 2.52^{\circ} \mathrm{C}\right)$ in summer season at site $\mathrm{V}$ and low values $\left(2.33 \pm 1.23^{\circ} \mathrm{C}\right)$ were recorded in winter at site I. pH ranged from $7.26( \pm 0.07)$ to $8.07( \pm 0.21)$ with the highest values in winter and the lowest in summer at most of the study sites. The $\mathrm{pH}$ values at the tail site (site $\mathrm{V}$ ) of the stream showed a decreased trend from wet to dry season while upstream values were higher during the dry season. DO values were generally higher at upstream sites and the lowest at the downstream sites. There was however a progressive 
TABLE 1: Physicochemical characteristics of water of Sukhnag stream (February 2011 to January 2012).

\begin{tabular}{|c|c|c|c|c|c|c|c|}
\hline S. no. & Parameters & Seasons & Site I & Site II & Site III & Site IV & Site V \\
\hline \multirow{8}{*}{1} & \multirow{8}{*}{ Water temperature $\left({ }^{\circ} \mathrm{C}\right)$} & Winter & $2.33 \pm 1.23$ & $3.00 \pm 0.87$ & $5.33 \pm 2.52$ & $5.83 \pm 3.82$ & $7.16 \pm 5.34$ \\
\hline & & Cvar.\% & 52.90 & 29.06 & 47.19 & 65.47 & 74.60 \\
\hline & & Spring & $10.63 \pm 3.50$ & $11.23 \pm 3.45$ & $12.46 \pm 3.55$ & $13.13 \pm 4.20$ & $14.50 \pm 5.27$ \\
\hline & & Cvar.\% & 32.91 & 30.70 & 28.48 & 31.99 & 36.33 \\
\hline & & Summer & $20.16 \pm 2.75$ & $21.80 \pm 1.80$ & $22.78 \pm 1.34$ & $23.80 \pm 1.47$ & $24.33 \pm 2.52$ \\
\hline & & Cvar.\% & 13.66 & 8.24 & 5.87 & 6.16 & 10.34 \\
\hline & & Autumn & $15.66 \pm 7.57$ & $16.70 \pm 7.15$ & $17.53 \pm 7.06$ & $18.10 \pm 6.77$ & $20.00 \pm 7.81$ \\
\hline & & Cvar.\% & 48.30 & 44.70 & 40.20 & 37.50 & 39.05 \\
\hline \multirow{8}{*}{2} & \multirow{8}{*}{ Depth (m) } & Winter & $0.50 \pm 0.25$ & $0.69 \pm 0.27$ & $0.97 \pm 0.37$ & $1.29 \pm 0.37$ & $1.38 \pm 0.77$ \\
\hline & & Cvar.\% & 49.64 & 38.60 & 38.34 & 60.32 & 55.53 \\
\hline & & Spring & $2.30 \pm 0.68$ & $2.50 \pm 0.40$ & $2.97 \pm 0.69$ & $2.90 \pm 0.41$ & $3.18 \pm 0.44$ \\
\hline & & Cvar.\% & 29.58 & 15.98 & 23.39 & 14.14 & 13.80 \\
\hline & & Summer & $1.08 \pm 0.05$ & $1.24 \pm 0.14$ & $1.61 \pm 0.64$ & $1.69 \pm 0.63$ & $1.40 \pm 0.04$ \\
\hline & & Cvar.\% & 4.65 & 10.83 & 39.82 & 37.47 & 3.03 \\
\hline & & Autumn & $1.10 \pm 0.10$ & $1.30 \pm 0.10$ & $1.55 \pm 0.22$ & $1.69 \pm 0.53$ & $1.93 \pm 0.64$ \\
\hline & & Cvar.\% & 9.09 & 7.69 & 14.17 & 31.08 & 33.25 \\
\hline \multirow{8}{*}{3} & \multirow{8}{*}{ Velocity $(\mathrm{m} / \mathrm{s})$} & Winter & $0.97 \pm 0.17$ & $0.93 \pm 0.16$ & $0.81 \pm 0.17$ & $0.38 \pm 0.04$ & $0.23 \pm 0.08$ \\
\hline & & Cvar.\% & 16.96 & 16.62 & 20.86 & 10.53 & 36.14 \\
\hline & & Spring & $1.51 \pm 0.20$ & $1.41 \pm 0.19$ & $1.09 \pm 0.14$ & $0.65 \pm 0.10$ & $0.39 \pm 0.03$ \\
\hline & & Cvar.\% & 13.47 & 13.34 & 12.84 & 15.33 & 7.24 \\
\hline & & Summer & $1.10 \pm 0.14$ & $1.01 \pm 0.17$ & $0.87 \pm 0.11$ & $0.54 \pm 0.06$ & $0.26 \pm 0.08$ \\
\hline & & Cvar.\% & 12.73 & 16.50 & 12.64 & 11.25 & 29.55 \\
\hline & & Autumn & $1.14 \pm 0.09$ & $1.07 \pm 0.05$ & $0.94 \pm 0.03$ & $0.37 \pm 0.06$ & $0.24 \pm 0.02$ \\
\hline & & Cvar.\% & 7.42 & 4.28 & 3.05 & 16.22 & 9.93 \\
\hline \multirow{8}{*}{4} & \multirow{8}{*}{$\mathrm{DO}(\mathrm{mg} / \mathrm{L})$} & Winter & $13.4 \pm 0.45$ & $12.4 \pm 0.23$ & $11.2 \pm 0.12$ & $10.1 \pm 0.06$ & $9.76 \pm 0.06$ \\
\hline & & Cvar.\% & 3.35 & 1.86 & 1.02 & 0.57 & 0.59 \\
\hline & & Spring & $11.90 \pm 0.70$ & $10.60 \pm 0.79$ & $9.63 \pm 0.60$ & $8.93 \pm 0.58$ & $8.33 \pm 0.55$ \\
\hline & & Cvar.\% & 5.89 & 7.49 & 6.26 & 6.46 & 6.61 \\
\hline & & Summer & $9.76 \pm 0.32$ & $8.83 \pm 0.47$ & $8.16 \pm 0.29$ & $7.06 \pm 0.31$ & $6.46 \pm 0.31$ \\
\hline & & Cvar.\% & 3.29 & 5.35 & 3.53 & 4.32 & 4.72 \\
\hline & & Autumn & $12.43 \pm 0.74$ & $11.40 \pm 0.66$ & $9.63 \pm 1.10$ & $8.26 \pm 1.01$ & $7.99 \pm 0.91$ \\
\hline & & Cvar.\% & 5.93 & 5.75 & 11.43 & 12.18 & 11.36 \\
\hline \multirow{8}{*}{5} & \multirow{8}{*}{ Free $\mathrm{CO}_{2}(\mathrm{mg} / \mathrm{L})$} & Winter & $6.50 \pm 0.26$ & $7.33 \pm 0.72$ & $7.63 \pm 0.64$ & $8.70 \pm 0.44$ & $9.00 \pm 0.36$ \\
\hline & & Cvar.\% & 4.07 & 9.86 & 8.32 & 5.01 & 4.01 \\
\hline & & Spring & $5.00 \pm 0.60$ & $7.16 \pm 0.76$ & $7.93 \pm 0.45$ & $10.06 \pm 1.00$ & $10.83 \pm 1.56$ \\
\hline & & Cvar.\% & 12.00 & 10.66 & 5.68 & 9.95 & 14.43 \\
\hline & & Summer & $4.43 \pm 0.75$ & $6.20 \pm 0.35$ & $9.26 \pm 0.81$ & $12.36 \pm 0.47$ & $13.36 \pm 0.68$ \\
\hline & & Cvar.\% & 16.93 & 5.59 & 8.72 & 3.82 & 5.09 \\
\hline & & Autumn & $5.76 \pm 0.32$ & $7.23 \pm 0.25$ & $8.66 \pm 0.56$ & $9.76 \pm 1.28$ & $10.23 \pm 1.05$ \\
\hline & & Cvar.\% & 5.57 & 3.48 & 6.56 & 13.21 & 10.26 \\
\hline \multirow{8}{*}{6} & \multirow{8}{*}{$\mathrm{pH}$} & Winter & $7.77 \pm 0.17$ & $7.67 \pm 0.04$ & $7.40 \pm 0.33$ & $7.53 \pm 0.02$ & $7.65 \pm 0.38$ \\
\hline & & Cvar.\% & 2.31 & 0.57 & 4.48 & 0.35 & 5.05 \\
\hline & & Spring & $7.83 \pm 0.11$ & $7.62 \pm 0.11$ & $7.46 \pm 0.15$ & $7.41 \pm 0.05$ & $7.37 \pm 0.07$ \\
\hline & & Cvar.\% & 1.47 & 1.46 & 2.05 & 0.68 & 1.03 \\
\hline & & Summer & $8.07 \pm 0.21$ & $7.56 \pm 0.32$ & $7.44 \pm 0.14$ & $7.34 \pm 0.03$ & $7.26 \pm 0.07$ \\
\hline & & Cvar.\% & 2.64 & 4.36 & 1.97 & 0.41 & 1.08 \\
\hline & & Autumn & $7.91 \pm 0.05$ & $7.69 \pm 0.01$ & $7.51 \pm 0.01$ & $7.51 \pm 0.08$ & $7.47 \pm 0.07$ \\
\hline & & Cvar.\% & 0.64 & 0.13 & 0.23 & 1.08 & 1.04 \\
\hline
\end{tabular}


TABle 1: Continued.

\begin{tabular}{|c|c|c|c|c|c|c|c|}
\hline S. no. & Parameters & Seasons & Site I & Site II & Site III & Site IV & Site V \\
\hline \multirow{8}{*}{7} & \multirow{8}{*}{ Alkalinity (mg/L) } & Winter & $74.66 \pm 8.14$ & $49.00 \pm 4.00$ & $52.66 \pm 4.16$ & $61.00 \pm 7.00$ & $67.33 \pm 4.50$ \\
\hline & & Cvar.\% & 8.16 & 7.91 & 11.48 & 6.70 & 10.91 \\
\hline & & Spring & $57.66 \pm 8.14$ & $63.33 \pm 7.57$ & $82.33 \pm 8.50$ & $90.33 \pm 5.50$ & $97.33 \pm 5.50$ \\
\hline & & Cvar.\% & 14.12 & 11.96 & 10.33 & 6.10 & 5.66 \\
\hline & & Summer & $76.00 \pm 4.58$ & $86.66 \pm 2.51$ & $94.00 \pm 2.64$ & $105.33 \pm 4.16$ & $114.33 \pm 3.21$ \\
\hline & & Cvar.\% & 6.03 & 2.90 & 2.81 & 3.95 & 2.81 \\
\hline & & Autumn & $66.00 \pm 8.18$ & $68.66 \pm 4.50$ & $75.00 \pm 6.55$ & $83.00 \pm 9.84$ & $93.33 \pm 6.02$ \\
\hline & & Cvar.\% & 12.40 & 6.57 & 8.74 & 11.87 & 6.46 \\
\hline \multirow{8}{*}{8} & \multirow{8}{*}{ Chloride (mg/L) } & Winter & $7.26 \pm 1.67$ & $8.56 \pm 0.58$ & $10.25 \pm 2.19$ & $12.30 \pm 1.84$ & $12.91 \pm 1.17$ \\
\hline & & Cvar.\% & 22.92 & 6.74 & 21.39 & 14.95 & 9.07 \\
\hline & & Spring & $3.03 \pm 0.32$ & $4.93 \pm 1.56$ & $13.90 \pm 1.20$ & $17.00 \pm 2.16$ & $18.00 \pm 2.00$ \\
\hline & & Cvar.\% & 10.60 & 31.68 & 8.63 & 12.73 & 11.08 \\
\hline & & Summer & $4.70 \pm 0.50$ & $5.36 \pm 0.45$ & $13.60 \pm 1.91$ & $14.40 \pm 1.64$ & $14.54 \pm 0.50$ \\
\hline & & Cvar.\% & 10.64 & 8.40 & 14.03 & 11.36 & 3.47 \\
\hline & & Autumn & $5.06 \pm 1.16$ & $7.00 \pm 0.70$ & $9.96 \pm 0.87$ & $13.20 \pm 1.31$ & $14.02 \pm 1.72$ \\
\hline & & Cvar.\% & 22.88 & 10.00 & 8.77 & 9.94 & 12.30 \\
\hline \multirow{8}{*}{9} & \multirow{8}{*}{ Conductivity $(\mu \mathrm{S} / \mathrm{cm})$} & Winter & $208.0 \pm 8.54$ & $235.6 \pm 43.59$ & $246.3 \pm 54.92$ & $287.0 \pm 38.3$ & $306.6 \pm 30.5$ \\
\hline & & Cvar.\% & 4.11 & 18.50 & 22.30 & 13.35 & 9.96 \\
\hline & & Spring & $269.3 \pm 19.6$ & $297.0 \pm 24.7$ & $263.3 \pm 44.8$ & $353.3 \pm 30.9$ & $396.6 \pm 20.8$ \\
\hline & & Cvar.\% & 7.28 & 8.34 & 17.04 & 8.77 & 5.25 \\
\hline & & Summer & $219.0 \pm 10.1$ & $239.6 \pm 31.0$ & $240.0 \pm 13.2$ & $260.0 \pm 14.8$ & $282.3 \pm 13.2$ \\
\hline & & Cvar.\% & 4.63 & 12.94 & 5.51 & 5.69 & 4.70 \\
\hline & & Autumn & $226.6 \pm 17.5$ & $239.0 \pm 32.0$ & $257.3 \pm 27.4$ & $280.3 \pm 17.3$ & $299.0 \pm 18.25$ \\
\hline & & Cvar.\% & 7.75 & 13.42 & 10.67 & 6.20 & 6.10 \\
\hline \multirow{8}{*}{10} & \multirow{8}{*}{ Total hardness (mg/L) } & Winter & $80.0 \pm 35.7$ & $101.3 \pm 49.5$ & $127.0 \pm 54.5$ & $141.6 \pm 59.1$ & $160.6 \pm 55.7$ \\
\hline & & Cvar.\% & 44.74 & 48.92 & 42.98 & 41.77 & 34.71 \\
\hline & & Spring & $129.3 \pm 12.5$ & $166.6 \pm 29.2$ & $179.3 \pm 26.86$ & $195.6 \pm 24.0$ & $257.0 \pm 33.81$ \\
\hline & & Cvar.\% & 9.67 & 17.57 & 14.98 & 12.27 & 13.15 \\
\hline & & Summer & $87.6 \pm 8.74$ & $101.6 \pm 15.5$ & $123.0 \pm 16.82$ & $138.6 \pm 21.5$ & $154.6 \pm 22.2$ \\
\hline & & Cvar.\% & 9.97 & 15.25 & 13.68 & 15.56 & 14.38 \\
\hline & & Autumn & $85.66 \pm 3.21$ & $100.3 \pm 7.64$ & $121.6 \pm 6.03$ & $138.3 \pm 12.4$ & $152.0 \pm 10.0$ \\
\hline & & Cvar.\% & 3.75 & 7.61 & 4.95 & 8.98 & 6.58 \\
\hline \multirow{8}{*}{11} & \multirow{8}{*}{ Calcium hardness (mg/L) } & Winter & $42.04 \pm 13.2$ & $51.8 \pm 18.08$ & $64.7 \pm 20.93$ & $74.75 \pm 18.4$ & $81.46 \pm 19.52$ \\
\hline & & Cvar.\% & 31.41 & 34.87 & 32.35 & 24.62 & 23.97 \\
\hline & & Spring & $70.38 \pm 4.44$ & $87.73 \pm 11.15$ & $98.36 \pm 4.32$ & $106.4 \pm 9.8$ & $115.3 \pm 11.0$ \\
\hline & & Cvar.\% & 6.31 & 12.71 & 4.39 & 9.25 & 9.55 \\
\hline & & Summer & $45.79 \pm 7.11$ & $56.07 \pm 9.53$ & $69.98 \pm 7.82$ & $81.18 \pm 11.24$ & $88.6 \pm 12.3$ \\
\hline & & Cvar.\% & 15.53 & 16.99 & 11.18 & 13.84 & 13.92 \\
\hline & & Autumn & $47.00 \pm 2.00$ & $57.33 \pm 9.07$ & $71.66 \pm 2.52$ & $84.00 \pm 7.55$ & $88.07 \pm 7.00$ \\
\hline & & Cvar.\% & 4.26 & 15.83 & 3.51 & 8.99 & 7.95 \\
\hline \multirow{8}{*}{12} & \multirow{8}{*}{ Magnesium hardness (mg/L) } & Winter & $9.22 \pm 5.74$ & $12.02 \pm 8.21$ & $15.13 \pm 8.53$ & $16.26 \pm 9.91$ & $19.24 \pm 8.81$ \\
\hline & & Cvar.\% & 62.25 & 68.26 & 56.33 & 60.94 & 45.77 \\
\hline & & Spring & $14.32 \pm 4.11$ & $19.18 \pm 8.30$ & $19.67 \pm 6.63$ & $21.67 \pm 5.97$ & $34.42 \pm 6.06$ \\
\hline & & Cvar.\% & 28.68 & 43.27 & 33.70 & 27.55 & 17.61 \\
\hline & & Summer & $10.17 \pm 1.54$ & $11.07 \pm 1.94$ & $12.88 \pm 3.04$ & $13.96 \pm 3.74$ & $16.03 \pm 2.63$ \\
\hline & & Cvar.\% & 15.17 & 17.51 & 23.60 & 26.75 & 16.39 \\
\hline & & Autumn & $9.39 \pm 0.37$ & $10.44 \pm 0.72$ & $12.15 \pm 1.26$ & $13.20 \pm 1.58$ & $15.53 \pm 0.72$ \\
\hline & & Cvar.\% & 3.95 & 6.98 & 10.39 & 11.97 & 4.70 \\
\hline
\end{tabular}


TABle 1: Continued.

\begin{tabular}{|c|c|c|c|c|c|c|c|}
\hline S. no. & Parameters & Seasons & Site I & Site II & Site III & Site IV & Site V \\
\hline \multirow{8}{*}{13} & \multirow{8}{*}{ Total solids $(\mathrm{mg} / \mathrm{L})$} & Winter & $139.3 \pm 40.7$ & $189.0 \pm 51.7$ & $214.6 \pm 56.8$ & $259.0 \pm 70.4$ & $265.6 \pm 71.4$ \\
\hline & & Cvar.\% & 29.23 & 27.37 & 26.50 & 27.18 & 26.90 \\
\hline & & Spring & $330.0 \pm 74.0$ & $400.0 \pm 68.9$ & $450.3 \pm 82.6$ & $487.0 \pm 93.3$ & $481.6 \pm 89.2$ \\
\hline & & Cvar.\% & 22.42 & 17.25 & 18.35 & 19.17 & 18.53 \\
\hline & & Summer & $180.3 \pm 31.5$ & $257.3 \pm 40.5$ & $298.0 \pm 33.4$ & $322.3 \pm 40.5$ & $321.0 \pm 40.5$ \\
\hline & & Cvar.\% & 17.50 & 15.74 & 11.22 & 12.58 & 12.62 \\
\hline & & Autumn & $165.3 \pm 10.0$ & $250.3 \pm 15.8$ & $300.0 \pm 19$ & $319.6 \pm 24.3$ & $320.0 \pm 22.6$ \\
\hline & & Cvar.\% & 6.06 & 6.32 & 6.33 & 7.63 & 7.06 \\
\hline \multirow{8}{*}{14} & \multirow{8}{*}{ TDS (mg/L) } & Winter & $116.6 \pm 27.3$ & $161.0 \pm 34.6$ & $178.6 \pm 35.0$ & $217.6 \pm 47.7$ & $233.6 \pm 48.9$ \\
\hline & & Cvar.\% & 23.40 & 21.49 & 19.60 & 21.94 & 20.95 \\
\hline & & Spring & $251.33 \pm 50.33$ & $295.0 \pm 39.1$ & $317.0 \pm 43.2$ & $341.3 \pm 50.21$ & $353.3 \pm 50.5$ \\
\hline & & Cvar.\% & 20.03 & 13.27 & 13.65 & 14.71 & 14.30 \\
\hline & & Summer & $143.3 \pm 17.2$ & $207.3 \pm 18.5$ & $231.6 \pm 15.5$ & $251.3 \pm 22.1$ & $262.0 \pm 27.8$ \\
\hline & & Cvar.\% & 12.03 & 8.96 & 6.71 & 8.80 & 10.63 \\
\hline & & Autumn & $139.0 \pm 4.0$ & $212.6 \pm 14.7$ & $241.6 \pm 17.6$ & $257.0 \pm 22.7$ & $266.0 \pm 20.4$ \\
\hline & & Cvar.\% & 2.88 & 6.93 & 7.29 & 8.84 & 7.68 \\
\hline \multirow{8}{*}{15} & \multirow{8}{*}{ TSS (mg/L) } & Winter & $22.66 \pm 13.42$ & $28.00 \pm 17.57$ & $36.00 \pm 22.86$ & $41.33 \pm 22.89$ & $32.00 \pm 22.86$ \\
\hline & & Cvar.\% & 59.24 & 62.78 & 63.53 & 55.40 & 71.47 \\
\hline & & Spring & $78.66 \pm 23.86$ & $105.00 \pm 29.86$ & $133.33 \pm 39.39$ & $145.66 \pm 43.13$ & $128.33 \pm 38.8$ \\
\hline & & Cvar.\% & 30.33 & 28.44 & 29.55 & 29.61 & 30.24 \\
\hline & & Summer & $37.00 \pm 14.52$ & $50.00 \pm 21.93$ & $66.33 \pm 17.89$ & $71.00 \pm 19$ & $59.00 \pm 13.45$ \\
\hline & & Cvar.\% & 39.26 & 43.86 & 26.98 & 26.76 & 22.80 \\
\hline & & Autumn & $26.33 \pm 6.02$ & $37.66 \pm 2.08$ & $58.33 \pm 1.52$ & $62.66 \pm 2.08$ & $54.00 \pm 2.64$ \\
\hline & & Cvar.\% & 22.89 & 5.53 & 2.62 & 3.32 & 4.90 \\
\hline \multirow{8}{*}{16} & \multirow{8}{*}{ Orthophosphate (mg/L) } & Winter & $0.03 \pm 0.01$ & $0.05 \pm 0.01$ & $0.06 \pm 0.01$ & $0.07 \pm 0.01$ & $0.09 \pm 0.02$ \\
\hline & & Cvar.\% & 21.53 & 20.22 & 20.31 & 19.52 & 25.01 \\
\hline & & Spring & $0.08 \pm 0.02$ & $0.10 \pm 0.02$ & $0.11 \pm 0.02$ & $0.11 \pm 0.02$ & $0.13 \pm 0.01$ \\
\hline & & Cvar.\% & 23.06 & 17.50 & 14.16 & 13.91 & 8.08 \\
\hline & & Summer & $0.04 \pm 0.03$ & $0.05 \pm 0.04$ & $0.06 \pm 0.05$ & $0.07 \pm 0.04$ & $0.07 \pm 0.04$ \\
\hline & & Cvar.\% & 67.53 & 72.64 & 69.58 & 62.45 & 58.12 \\
\hline & & Autumn & $0.03 \pm 0.01$ & $0.04 \pm 0.01$ & $0.05 \pm 0.01$ & $0.06 \pm 0.01$ & $0.07 \pm 0.01$ \\
\hline & & Cvar.\% & 20.59 & 25.65 & 19.67 & 20.69 & 18.18 \\
\hline \multirow{8}{*}{17} & \multirow{8}{*}{ Total phosphorus (mg/L) } & Winter & $0.18 \pm 0.02$ & $0.19 \pm 0.03$ & $0.20 \pm 0.03$ & $0.27 \pm 0.05$ & $0.29 \pm 0.05$ \\
\hline & & Cvar.\% & 11.96 & 14.25 & 13.57 & 17.23 & 17.62 \\
\hline & & Spring & $0.23 \pm 0.02$ & $0.28 \pm 0.03$ & $0.28 \pm 0.01$ & $0.38 \pm 0.01$ & $0.41 \pm 0.02$ \\
\hline & & Cvar.\% & 7.93 & 11.41 & 2.81 & 1.59 & 4.82 \\
\hline & & Summer & $0.18 \pm 0.02$ & $0.20 \pm 0.04$ & $0.22 \pm 0.04$ & $0.32 \pm 0.03$ & $0.33 \pm 0.03$ \\
\hline & & Cvar.\% & 11.32 & 19.64 & 16.54 & 9.34 & 10.07 \\
\hline & & Autumn & $0.17 \pm 0.01$ & $0.18 \pm 0.01$ & $0.20 \pm 0.01$ & $0.3 \pm 0.01$ & $0.32 \pm 0.01$ \\
\hline & & Cvar.\% & 3.08 & 3.32 & 2.81 & 3.05 & 2.38 \\
\hline \multirow{8}{*}{18} & \multirow{8}{*}{ Ammoniacal nitrogen (mg/L) } & Winter & $0.08 \pm 0.02$ & $0.11 \pm 0.04$ & $0.13 \pm 0.04$ & $0.15 \pm 0.04$ & $0.16 \pm 0.05$ \\
\hline & & Cvar.\% & 25.81 & 30.81 & 32.31 & 29.09 & 26.67 \\
\hline & & Spring & $0.04 \pm 0.01$ & $0.05 \pm 0.01$ & $0.05 \pm 0.01$ & $0.06 \pm 0.02$ & $0.07 \pm 0.02$ \\
\hline & & Cvar.\% & 13.01 & 18.34 & 22.57 & 26.53 & 29.42 \\
\hline & & Summer & $0.03 \pm 0.00$ & $0.04 \pm 0.00$ & $0.04 \pm 0.01$ & $0.05 \pm 0.00$ & $0.06 \pm 0.01$ \\
\hline & & Cvar.\% & 4.06 & 9.61 & 10.42 & 7.91 & 7.94 \\
\hline & & Autumn & $0.05 \pm 0.02$ & $0.07 \pm 0.038$ & $0.09 \pm 0.03$ & $0.09 \pm 0.03$ & $0.10 \pm 0.04$ \\
\hline & & Cvar.\% & 42.04 & 48.91 & 43.26 & 41.12 & 45.92 \\
\hline
\end{tabular}


TABle 1: Continued.

\begin{tabular}{|c|c|c|c|c|c|c|c|}
\hline S. no. & Parameters & Seasons & Site I & Site II & Site III & Site IV & Site V \\
\hline \multirow{8}{*}{19} & \multirow{8}{*}{ Nitrite-nitrogen (mg/L) } & Winter & $0.007 \pm 0.00$ & $0.01 \pm 0.00$ & $0.01 \pm 0.00$ & $0.01 \pm 0.00$ & $0.01 \pm 0.00$ \\
\hline & & Cvar.\% & 30.12 & 20.15 & 22.43 & 19.50 & 29.61 \\
\hline & & Spring & $0.009 \pm 0.003$ & $0.01 \pm 0.003$ & $0.017 \pm 0.0$ & $0.019 \pm 0.0$ & $0.021 \pm 0.0$ \\
\hline & & Cvar.\% & 33.25 & 30.70 & 29.04 & 18.16 & 14.29 \\
\hline & & Summer & $0.028 \pm 0.004$ & $0.03 \pm 0.007$ & $0.04 \pm 0.004$ & $0.04 \pm 0.008$ & $0.06 \pm 0.01$ \\
\hline & & Cvar.\% & 14.69 & 20.46 & 9.52 & 16.70 & 16.64 \\
\hline & & Autumn & $0.016 \pm 0.005$ & $0.018 \pm 0.006$ & $0.02 \pm 0.012$ & $0.021 \pm 0.005$ & $0.02 \pm 0.01$ \\
\hline & & Cvar.\% & 35.87 & 37.13 & 52.83 & 23.59 & 34.48 \\
\hline \multirow{8}{*}{20} & \multirow{8}{*}{ Nitrate-nitrogen (mg/L) } & Winter & $0.26 \pm 0.02$ & $0.39 \pm 0.04$ & $0.47 \pm 0.07$ & $0.50 \pm 0.10$ & $0.51 \pm 0.10$ \\
\hline & & Cvar.\% & 8.44 & 10.47 & 16.15 & 19.91 & 19.42 \\
\hline & & Spring & $0.35 \pm 0.04$ & $0.51 \pm 0.10$ & $0.68 \pm 0.06$ & $0.76 \pm 0.03$ & $0.78 \pm 0.05$ \\
\hline & & Cvar.\% & 12.20 & 21.03 & 9.19 & 5.16 & 6.37 \\
\hline & & Summer & $0.30 \pm 0.02$ & $0.44 \pm 0.06$ & $0.51 \pm 0.09$ & $0.56 \pm 0.09$ & $0.58 \pm 0.10$ \\
\hline & & Cvar.\% & 8.08 & 14.00 & 17.48 & 17.33 & 18.42 \\
\hline & & Autumn & $0.29 \pm 0.02$ & $0.44 \pm 0.04$ & $0.49 \pm 0.01$ & $0.52 \pm 0.02$ & $0.53 \pm 0.01$ \\
\hline & & Cvar.\% & 7.14 & 9.30 & 2.35 & 3.87 & 3.51 \\
\hline \multirow{8}{*}{21} & \multirow{8}{*}{ Organic nitrogen $(\mathrm{mg} / \mathrm{L})$} & Winter & $0.13 \pm 0.01$ & $0.19 \pm 0.01$ & $0.21 \pm 0.01$ & $0.23 \pm 0.02$ & $0.25 \pm 0.03$ \\
\hline & & Cvar.\% & 7.64 & 4.61 & 3.96 & 6.61 & 10.87 \\
\hline & & Spring & $0.20 \pm 0.03$ & $0.27 \pm 0.03$ & $0.29 \pm 0.03$ & $0.31 \pm 0.03$ & $0.35 \pm 0.04$ \\
\hline & & Cvar.\% & 15.08 & 10.78 & 10.59 & 11.11 & 12.02 \\
\hline & & Summer & $0.29 \pm 0.01$ & $0.39 \pm 0.01$ & $0.48 \pm 0.03$ & $0.55 \pm 0.04$ & $0.62 \pm 0.06$ \\
\hline & & Cvar.\% & 3.59 & 3.26 & 6.66 & 6.57 & 9.38 \\
\hline & & Autumn & $0.19 \pm 0.03$ & $0.29 \pm 0.05$ & $0.33 \pm 0.06$ & $0.35 \pm 0.06$ & $0.38 \pm 0.09$ \\
\hline & & Cvar.\% & 13.64 & 17.10 & 19.03 & 16.41 & 22.25 \\
\hline \multirow{8}{*}{22} & \multirow{8}{*}{ Total nitrogen (mg/L) } & Winter & $0.49 \pm 0.01$ & $0.71 \pm 0.07$ & $0.83 \pm 0.05$ & $0.91 \pm 0.07$ & $0.95 \pm 0.08$ \\
\hline & & Cvar.\% & 1.06 & 9.53 & 6.57 & 7.93 & 8.39 \\
\hline & & Spring & $0.60 \pm 0.07$ & $0.87 \pm 0.13$ & $1.05 \pm 0.08$ & $1.16 \pm 0.05$ & $1.23 \pm 0.07$ \\
\hline & & Cvar.\% & 11.11 & 15.04 & 7.39 & 4.69 & 5.47 \\
\hline & & Summer & $0.66 \pm 0.03$ & $0.92 \pm 0.07$ & $1.09 \pm 0.08$ & $1.22 \pm 0.11$ & $1.34 \pm 0.14$ \\
\hline & & Cvar.\% & 5.07 & 7.31 & 7.08 & 8.65 & 10.58 \\
\hline & & Autumn & $0.56 \pm 0.02$ & $0.83 \pm 0.02$ & $0.93 \pm 0.04$ & $0.99 \pm 0.02$ & $1.06 \pm 0.04$ \\
\hline & & Cvar.\% & 3.75 & 1.86 & 4.28 & 1.61 & 3.74 \\
\hline \multirow{8}{*}{23} & \multirow{8}{*}{ Calcium ion $(\mathrm{mg} / \mathrm{L})$} & Winter & $16.83 \pm 5.28$ & $20.77 \pm 7.24$ & $25.91 \pm 8.38$ & $29.93 \pm 7.34$ & $32.62 \pm 7.81$ \\
\hline & & Cvar.\% & 31.41 & 34.87 & 32.35 & 24.62 & 23.97 \\
\hline & & Spring & $28.18 \pm 1.77$ & $35.13 \pm 4.46$ & $39.39 \pm 1.72$ & $42.64 \pm 3.94$ & $46.18 \pm 4.41$ \\
\hline & & Cvar.\% & 6.31 & 12.71 & 4.39 & 9.25 & 9.55 \\
\hline & & Summer & $21.22 \pm 7.84$ & $25.98 \pm 9.68$ & $32.24 \pm 10.43$ & $37.53 \pm 13.15$ & $40.79 \pm 13.18$ \\
\hline & & Cvar.\% & 36.94 & 37.29 & 32.36 & 35.05 & 32.32 \\
\hline & & Autumn & $18.82 \pm 0.8$ & $22.96 \pm 3.63$ & $28.7 \pm 1.0$ & $33.64 \pm 3.02$ & $35.27 \pm 2.80$ \\
\hline & & Cvar.\% & 4.26 & 15.83 & 3.51 & 8.99 & 7.95 \\
\hline \multirow{8}{*}{24} & \multirow{8}{*}{ Magnesium ion (mg/L) } & Winter & $3.91 \pm 1.22$ & $4.83 \pm 1.68$ & $6.02 \pm 1.94$ & $6.96 \pm 1.71$ & $7.58 \pm 1.81$ \\
\hline & & Cvar.\% & 31.41 & 34.87 & 32.35 & 24.62 & 23.97 \\
\hline & & Spring & $5.99 \pm 0.38$ & $7.47 \pm 0.95$ & $8.38 \pm 0.36$ & $9.07 \pm 0.83$ & $9.82 \pm 0.93$ \\
\hline & & Cvar.\% & 6.31 & 12.71 & 4.39 & 9.25 & 9.55 \\
\hline & & Summer & $4.96 \pm 1.94$ & $6.08 \pm 2.42$ & $7.53 \pm 2.61$ & $8.78 \pm 3.28$ & $9.55 \pm 3.33$ \\
\hline & & Cvar.\% & 39.21 & 39.77 & 34.69 & 37.43 & 34.96 \\
\hline & & Autumn & $4.18 \pm 0.177$ & $5.10 \pm 0.80$ & $6.37 \pm 0.22$ & $7.47 \pm 0.67$ & $7.83 \pm 0.62$ \\
\hline & & Cvar.\% & 4.26 & 15.83 & 3.51 & 8.99 & 7.95 \\
\hline
\end{tabular}


TABle 1: Continued.

\begin{tabular}{|c|c|c|c|c|c|c|c|}
\hline S. no. & Parameters & Seasons & Site I & Site II & Site III & Site IV & Site V \\
\hline \multirow{8}{*}{25} & \multirow{8}{*}{ Sodium (mg/L) } & Winter & $6.23 \pm 1.95$ & $7.69 \pm 2.68$ & $9.59 \pm 3.10$ & $11.08 \pm 2.72$ & $12.08 \pm 2.89$ \\
\hline & & Cvar.\% & 31.41 & 34.87 & 32.35 & 24.62 & 23.97 \\
\hline & & Spring & $9.39 \pm 0.59$ & $11.71 \pm 1.48$ & $13.13 \pm 0.57$ & $14.21 \pm 1.31$ & $15.39 \pm 1.47$ \\
\hline & & Cvar.\% & 6.31 & 12.71 & 4.39 & 9.25 & 9.55 \\
\hline & & Summer & $7.20 \pm 2.50$ & $8.81 \pm 3.09$ & $10.94 \pm 3.30$ & $12.74 \pm 4.18$ & $13.85 \pm 4.19$ \\
\hline & & Cvar.\% & 34.75 & 35.15 & 30.18 & 32.87 & 30.26 \\
\hline & & Autumn & $6.49 \pm 0.27$ & $7.91 \pm 1.25$ & $9.89 \pm 0.34$ & $11.6 \pm 1.04$ & $12.16 \pm 0.96$ \\
\hline & & Cvar.\% & 4.26 & 15.83 & 3.51 & 8.99 & 7.95 \\
\hline \multirow{8}{*}{26} & \multirow{8}{*}{ Potassium (mg/L) } & Winter & $1.55 \pm 0.48$ & $1.92 \pm 0.67$ & $2.39 \pm 0.77$ & $2.77 \pm 0.68$ & $3.02 \pm 0.72$ \\
\hline & & Cvar.\% & 31.41 & 34.87 & 32.35 & 24.62 & 23.97 \\
\hline & & Spring & $2.18 \pm 0.14$ & $2.70 \pm 0.22$ & $3.05 \pm 0.29$ & $3.29 \pm 0.32$ & $3.56 \pm 0.26$ \\
\hline & & Cvar.\% & 6.55 & 8.48 & 9.60 & 9.96 & 7.49 \\
\hline & & Summer & $1.56 \pm 0.43$ & $1.90 \pm 0.49$ & $2.37 \pm 0.56$ & $2.76 \pm 0.70$ & $2.98 \pm 0.62$ \\
\hline & & Cvar.\% & 28.08 & 26.28 & 23.79 & 25.56 & 20.84 \\
\hline & & Autumn & $1.70 \pm 0.07$ & $2.08 \pm 0.32$ & $2.60 \pm 0.09$ & $3.05 \pm 0.27$ & $3.20 \pm 0.25$ \\
\hline & & Cvar.\% & 4.26 & 15.83 & 3.51 & 8.99 & 7.95 \\
\hline
\end{tabular}

Cvar.\%: Percentage Co-variance.

increase in DO $(6.46 \pm 0.31$ to $13.4 \pm 0.45)$ at all the sampling sites during the transition to rainy, winter season. Variation in $\mathrm{EC}$ was significant $(\mathrm{CV}=5.2-18.5 \%, P<0.01)$ among seasons and at all sampling sites $(P<0.05)$. Higher values of EC were recorded in spring $(396.6 \pm 20.8 \mu \mathrm{S} / \mathrm{cm})$ at the tail site $($ site V) and lower in winter $(208.0 \pm 8.54 \mu \mathrm{S} / \mathrm{cm})$ at the headstream site (site I). The higher EC is attributed to the high degree of anthropogenic activities such as waste disposal and agricultural runoff. The seasonal variations in depth during one year of study showed that it was highest in the spring season. By autumn the depth starts to decrease and is lowest in the winter. The water depth at all the sites varied both spatially $(P<0.05)$ and temporally $(\mathrm{CV}=4.6-60.3 \%$, $P<0.01)$. Maximum surface water velocities for the five sites were recorded in spring season (peak flow season) and minimum were recorded in winter season (snowy season). Surface water velocities showed greater variability in the winter season $(\mathrm{CV}=10.5-36.1 \%)$ than in autumn season $(\mathrm{CV}=4.2-16.2 \%)$. Ortho-phosphorus and total phosphorus concentrations were highest $(0.13 \pm 0.01 \mathrm{mg} / \mathrm{L}$ and $0.41 \pm$ $0.02 \mathrm{mg} / \mathrm{L}$, resp. $)$ in spring at site $\mathrm{V}$ and lowest $(0.03 \pm 0.01$ and $0.17 \pm 0.01 \mathrm{mg} / \mathrm{L}$, resp.) in autumn at site I. Concentrations of $\mathrm{NO}_{3}-\mathrm{N}$ and $\mathrm{NO}_{2}-\mathrm{N}$ were highest $(0.78 \pm 0.05$ and $0.021 \pm$ $0.0 \mathrm{mg} / \mathrm{L}$, resp.) in summer at site $\mathrm{V}$ and lowest $(0.26 \pm 0.02$ and $0.007 \pm 0.00 \mathrm{mg} / \mathrm{L}$, resp.) in winter at site I. Ammonical$\mathrm{N}$ showed the highest values in winter at site $\mathrm{V}(0.16 \pm$ $0.05)$ and lowest values in summer at site $\mathrm{I}(0.03 \pm 0.00)$. The highest values of organic- $\mathrm{N}$ and total nitrogen $(0.62 \pm$ 0.06 and $1.34 \pm 0.14 \mathrm{mg} / \mathrm{L}$, resp.) were found in summer at site $\mathrm{V}$ and lowest $(0.13 \pm 0.01$ and $0.49 \pm 0.01 \mathrm{mg} / \mathrm{L}$, resp.) in winter at site I. Total hardness, calcium hardness, and magnesium hardness were observed highest in spring and lowest in autumn and winter at all the sites. Lower levels of total hardness, calcium hardness, and magnesium hardness were observed at upstream sites compared to the downstream sites and among the seasons; the lowest values were recorded in the winter at all the sites. The highest values of $\mathrm{Ca}^{2+}, \mathrm{Mg}^{2+}$, $\mathrm{Na}^{+}, \mathrm{K}^{+}$, and TDS $(46.18 \pm 4.41,9.82 \pm 0.93,15.39 \pm 1.47,3.56$ \pm 0.26 , and $353.3 \pm 50.5 \mathrm{mg} / \mathrm{L}$, resp.) were recorded at the tail site (site V) in spring season and lowest $(16.83 \pm 5.28,3.91$ $\pm 1.22,6.23 \pm 1.95,1.55 \pm 0.48$, and $116.6 \pm 27.3 \mathrm{mg} / \mathrm{L}$, resp.) at the headstream site (site I) in the winter season. TDS and TSS values were recorded highest $(487.0 \pm 93.3$ and $145.66 \pm$ $43.13 \mathrm{mg} / \mathrm{L}$, resp.) in spring at site IV and lowest $(139.3 \pm 40.7$ and $22.66 \pm 13.42 \mathrm{mg} / \mathrm{L}$, resp.) in winter at site I. The overall values of CV showed significant difference of concentrations from head to tail. On the basis of molar concentrations, among the cations, $\mathrm{Ca}^{2+}$ and $\mathrm{Na}^{+}$were dominant and $\mathrm{Mg}^{+2}$ and $\mathrm{K}^{+}$were found in minor concentrations. Chloride was the dominant anion observed. Overall we observed significant degree of spatial and temporal variations in the concentration of water quality parameters using ANOVA $(P<0.05)$ and $t$ test $(P<0.01)$ analysis.

Domestic wastewaters, particularly those containing detergents and fertilizer runoff, contribute to the higher levels of phosphates in the water column. Phosphate concentrations indicate the presence of anthropogenic pollutants [35]. The nitrate- $\mathrm{N}$ and organic nitrogen concentrations had spatial distributions that increased from the upstream to downstream, mainly due to the contributions of agricultural runoff and sewage discharge [36].

The accumulation factor (AF) and river recovery capacity (RRC) of the physicochemical parameters during the sampling period are presented in Table 2. Accumulation factor of the parameters revealed that the nitrate and nitrite of downstream water were about 2.2 and 2.9 times, respectively, more than what was observed upstream. Other parameters showed an average accumulation factor of 1.6. Chloride, $\mathrm{NO}_{3}-\mathrm{N}, \mathrm{NO}_{2}-\mathrm{N}$, and free $\mathrm{CO}_{2}$ showed the highest percentage recoveries of about $66 \%, 50 \%, 51 \%$, and $50 \%$, respectively, while conductivity showed the lowest recovery of about $28 \%$ in water downstream. Recovery values for 


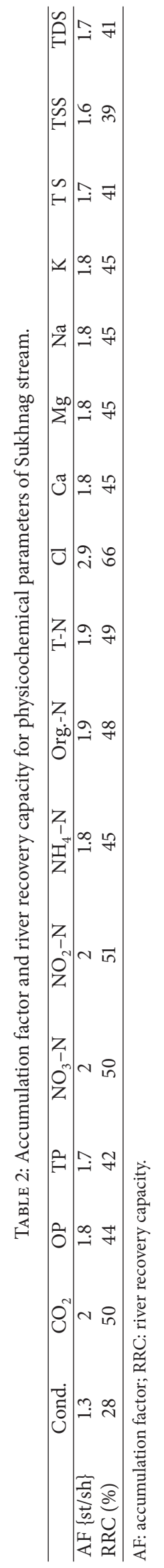


water quality parameters indicated that there was little or no change in values at the tail site compared to headstream site. The accumulation factor of the physicochemical parameters clearly indicated higher values downstream compared to the reference point upstream and is a clear indication of anthropogenic impact. The low recovery values observed for most of the parameters suggest that these substances are being released into the river in quantities that exceed the removal carrying capacity of the Sukhnag stream [37]. The higher levels of these nutrients clearly surpass the river recovery capacity.

3.1. Regression Analysis (RA). To explain the nature and magnitude of relationships among various physicochemical parameters, we plotted concentrations of all dependent variables against independent variables. The observed relationships between dependent variable and independent variable concentrations $[\log (X)]$ were different and not significant for all parameters. Concentrations of most variables increased with increasing independent variable (Table 3 ). The results of the statistical analysis with the general linear regression model (Figure 2) showed strong significant positive relationships $(P<0.0001)$ of water temperature with organic-N, free $\mathrm{CO}_{2}$, and $\mathrm{NO}_{2}-\mathrm{N}$; depth with velocity, Total-P, $\mathrm{NO}_{3}-\mathrm{N}$, TS, TSS, and TDS; conductivity with total hardness; velocity with total-P, $\mathrm{NO}_{3}-\mathrm{N}$, TS, TSS, and TDS; total-P with $\mathrm{NO}_{3}-\mathrm{N}$, $\mathrm{Ca}^{2+}$ and TS; alkalinity with $\mathrm{NO}_{3}-\mathrm{N}$, organic- $\mathrm{N}$, and $\mathrm{NO}_{2}-$ $\mathrm{N}$; TDS with total-P and $\mathrm{NO}_{3}-\mathrm{N}$, while strong significant negative $(P<0.0001)$ relationship was shown by water temperature with $\mathrm{DO}$; DO with free $\mathrm{CO}_{2}$ and organic-N. Concentrations of $\mathrm{NH}_{4}-\mathrm{N}$ and alkalinity respond moderately to water temperature and $\mathrm{DO}$, respectively. Concentration of $\mathrm{K}^{+}, \mathrm{Mg}^{2+}$, and $\mathrm{Cl}^{-}$showed reasonable relationship with stream flow. Other variables have positive relationships as shown in Table 3.

Depth increases with higher runoff, which in turn brings higher load of nitrate from this agriculture dominated watershed in spring and summer seasons. Nitrate is more associated with the use of organic and inorganic fertilizers [38, 39]. Significant variation of total-P with depth and velocity could be due to the agricultural activity since farmers use phosphate as a fertilizer. The relation of suspended solids with depth and velocity indicated agricultural runoff. The linear curve fitting model of both minerals and nutrients reflected that their origin in river runoff is from the agricultural field along with waste disposal activity.

3.2. Cluster Analysis (CA). The cluster analysis is useful in solving classification problems, whose objective is to place factors or variables into groups such that the degree of association is strong between members of the same cluster and weak between members of different clusters [40]. In this study, CA showed strong spatial and temporal association on the basis of variations of principal pollution factors and indicated that the effects of human activities on water quality vary spatially as well as temporally. The dendrogram indicates pollution status as well as the effect of contamination at the sampling sites. It provides a visual summary of the clustering processes, presenting a picture of the groups and their proximity. Cluster analysis (CA) was used to detect similarity between the five sampling sites and four seasons. CA generated a dendrogram, grouping the sampling sites and the months on the basis of percentage of similarity and dissimilarity of water quality parameters. The dendrogram of percentage similarity of five study sites, on the basis of physicochemical factors, is presented in Figure 3. The analysis of similarity of study sites from $82 \%$ to $100 \%$ was carried out to indicate relationship intensity between sites as cluster. The Bray-Curtis similarity analysis confirmed that there is a similarity of $96 \%$ between sites IV and V and 94\% between sites II and III. Contrary to these sites, site I showed maximum dissimilarity with other sites during the entire study period as it is located on the head portion of the stream. Hence, the impact of human activities on the stream ecosystem at site I is relatively low. The dendrogram of percentage seasonal similarity (Figure 4) shows that there is a maximum similarity of $97 \%$ between spring and autumn and $82 \%$ between winter and summer clusters. Summer and winter clusters showed only $82 \%$ similarity with spring and autumn clusters. The generated dendrogram grouped the sampling sites and seasons into three groups. Using this analysis we could categorize study sites into three groups: low pollution (site I), moderate pollution (sites II and III) and high pollution (sites IV and V). Seasonal grouping showed higher inorganic and organic loads during spring and autumn seasons.

3.3. Principal Component Analysis (PCA). Principal component analysis was carried out to extract the most important factors and physicochemical parameters affecting the water quality. Due to the complex relationships, it was difficult to draw clear conclusions. However, not only could principal component analysis extract the information to some extent and explain the structure of the data in detail, on temporal characteristics by clustering the samples, but it could also describe their different characteristics and help to elucidate the relationship between different variables by the variable lines. SPSS 16.0 and PAST software were used to carry out principal component analysis to determine the main principal components from the original variables [41-43]. Based on the eigenvalues screen plot (Figure 5), the 26 physicochemical parameters were reduced to 2 main factors (factors 1 and 2) from the leveling off point(s) in the screen plot [44]. The first factor corresponding to the largest eigenvalue (17.16) accounts for approximately $66.00 \%$ of the total variance. The second factor corresponding to the second eigenvalue (7.96) accounts for approximately $30.63 \%$ of the total variance. The remaining 24 factors have eigenvalues of less than unity. Any factor with an eigenvalue greater than 1 is considered significant $[44,45]$. A correlation matrix of these variables was computed, and factor loading was defined to explore the nature of variation and principal patterns among them [46]. Further analysis of factor loadings showed that velocity, total$\mathrm{P}, \mathrm{NO}_{3}-\mathrm{N}, \mathrm{NO}_{2}-\mathrm{N}$, organic- $\mathrm{N}, \mathrm{Ca}^{2+}, \mathrm{Na}^{+}, \mathrm{TS}, \mathrm{TSS}, \mathrm{TDS}$, and free $\mathrm{CO}_{2}$ were the 11 major factors affecting the water quality of Sukhnag stream (Table 4). For factor 1, velocity, total$\mathrm{P}, \mathrm{NO}_{3}-\mathrm{N}, \mathrm{Ca}^{2+}, \mathrm{Na}^{+}$, TS, TSS, and TDS have the highest factor loading value $(>0.96)$ and showed that these are the most influential variables for the first factor or principal 


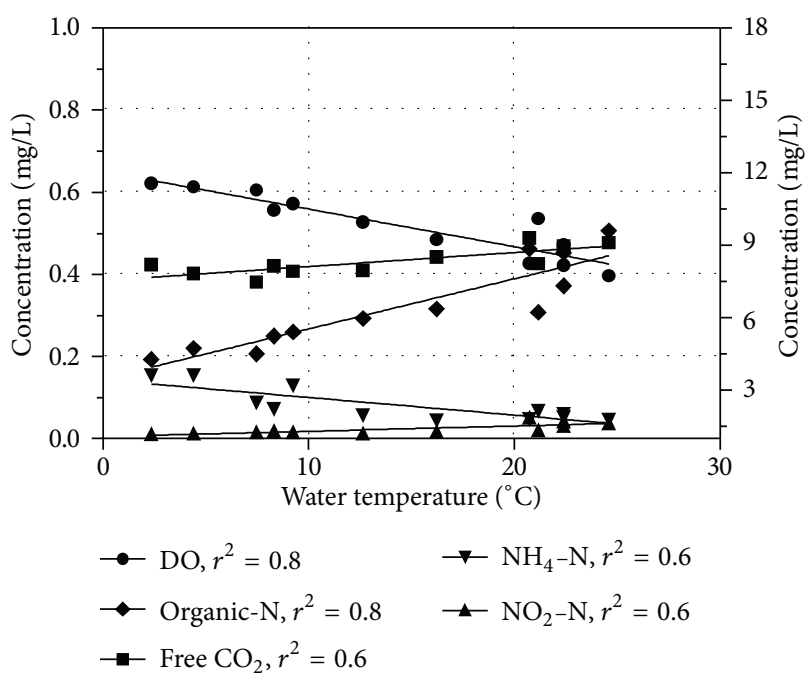

(a)

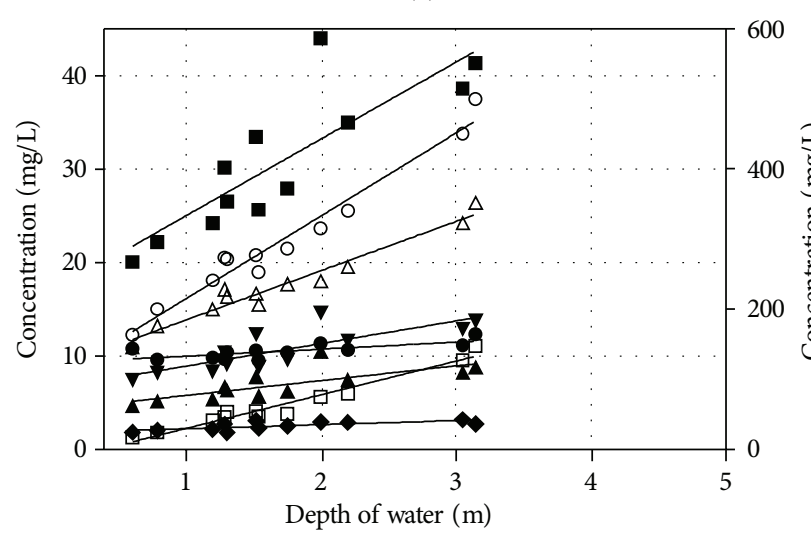

$$
\begin{array}{ll}
\rightarrow-\mathrm{Ca}^{2+}, r^{2}=0.7 & \rightarrow \mathrm{Na}^{+}, r^{2}=0.6 \\
-\mathrm{TS}, r^{2}=0.9 & \rightarrow \mathrm{Mg}^{2+}, r^{2}=0.5 \\
\triangle \mathrm{TDS}, r^{2}=0.9 & \rightarrow-\mathrm{TSS}, r^{2}=0.9 \\
\rightarrow \mathrm{Cl}^{-}, r^{2}=0.5 & \rightarrow \mathrm{K}^{+}, r^{2}=0.5
\end{array}
$$

(c)

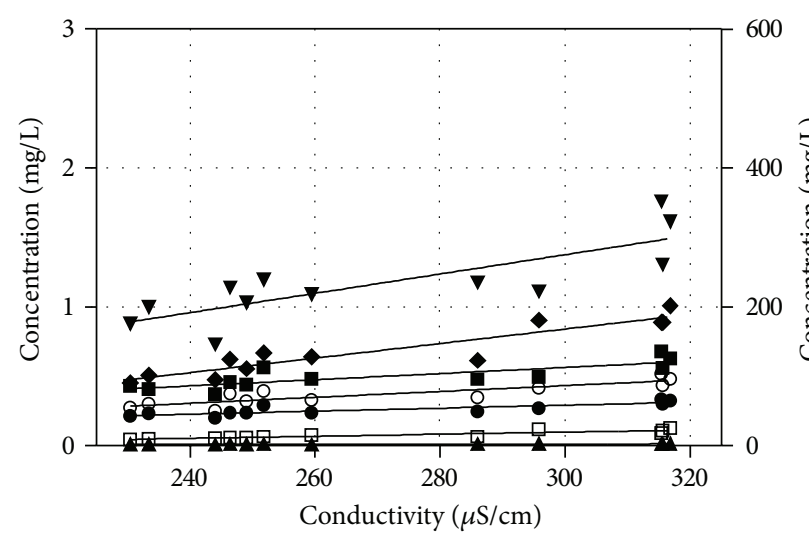

- Total-P, $r^{2}=0.7$

$\checkmark$ T hardness, $r^{2}=0.8$

$-\mathrm{NO}_{3}-\mathrm{N}, r^{2}=0.6$

- Ca hardness, $r^{2}=0.7$

$\leftarrow \mathrm{K}^{+}, r^{2}=0.4$

$\square$ Mg hardness, $r^{2}=0.7$

$\rightarrow$ TDS, $r^{2}=0.6$

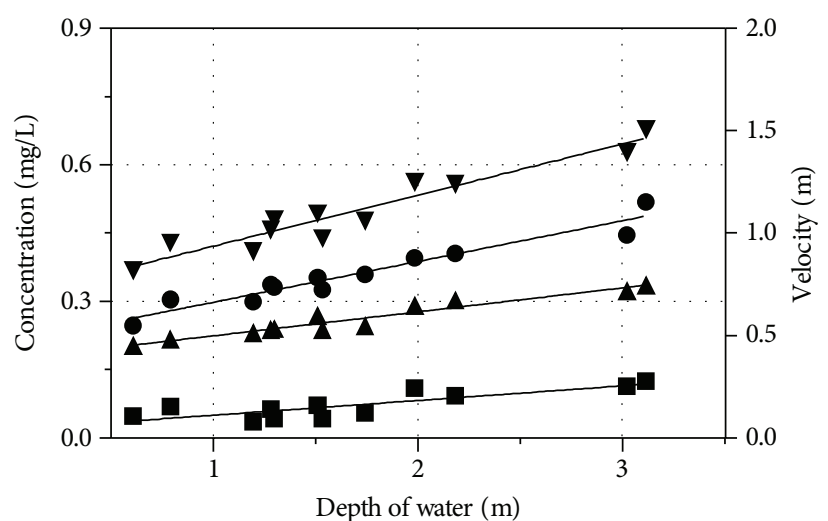

$$
\begin{aligned}
& \longrightarrow \text { Velocity, } r^{2}=0.9 \\
& \smile \text { Total-P, } r^{2}=0.9 \quad \longrightarrow \mathrm{NO}_{3}-\mathrm{N}, r^{2}=0.9
\end{aligned}
$$

(b)

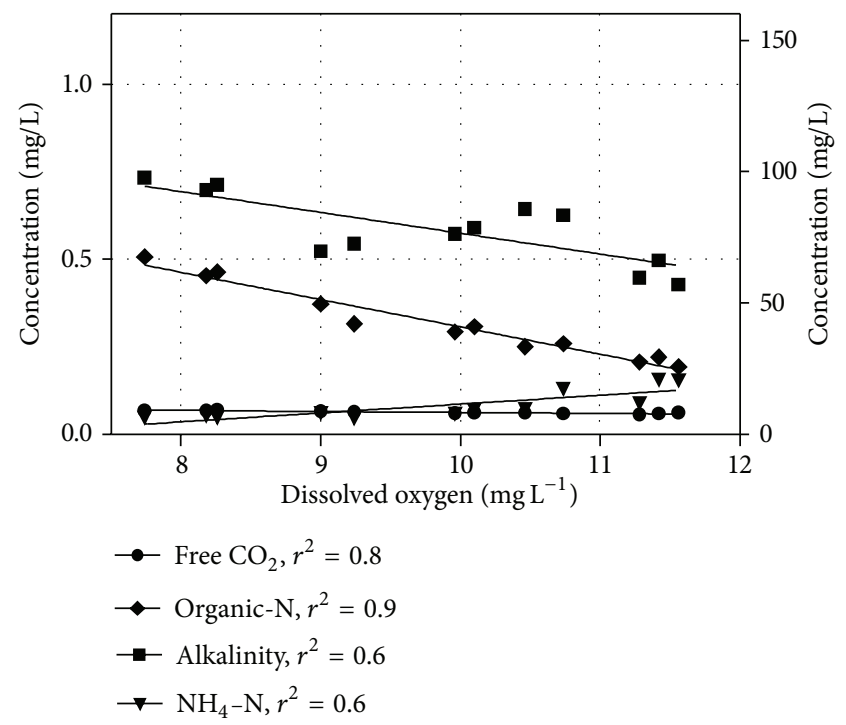

(d)

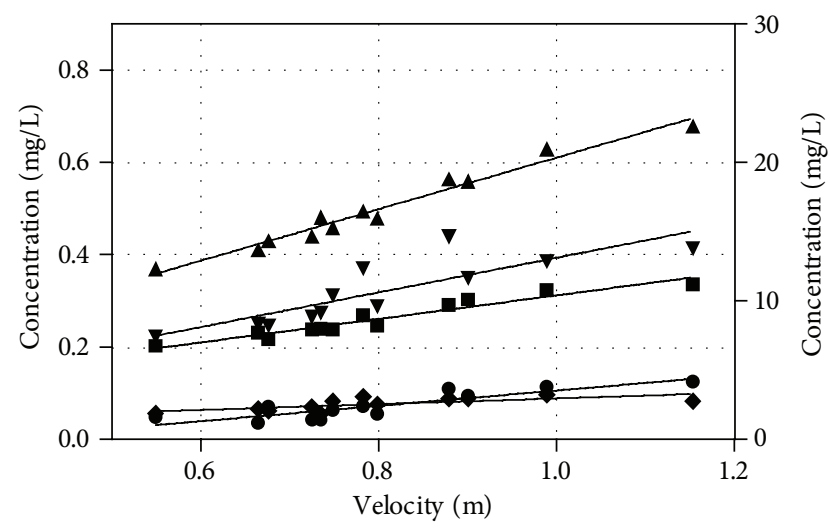

$$
\begin{aligned}
& \longrightarrow \text { Ortho-P, } r^{2}=0.7 \rightarrow \mathrm{Na}^{+}, r^{2}=0.7 \\
& \rightarrow \text { Total-P, } r^{2}=0.9 \quad \longrightarrow \mathrm{K}^{+}, r^{2}=0.4 \\
& \leftarrow \mathrm{NO}_{3}-\mathrm{N}, r^{2}=0.9
\end{aligned}
$$

(e) 

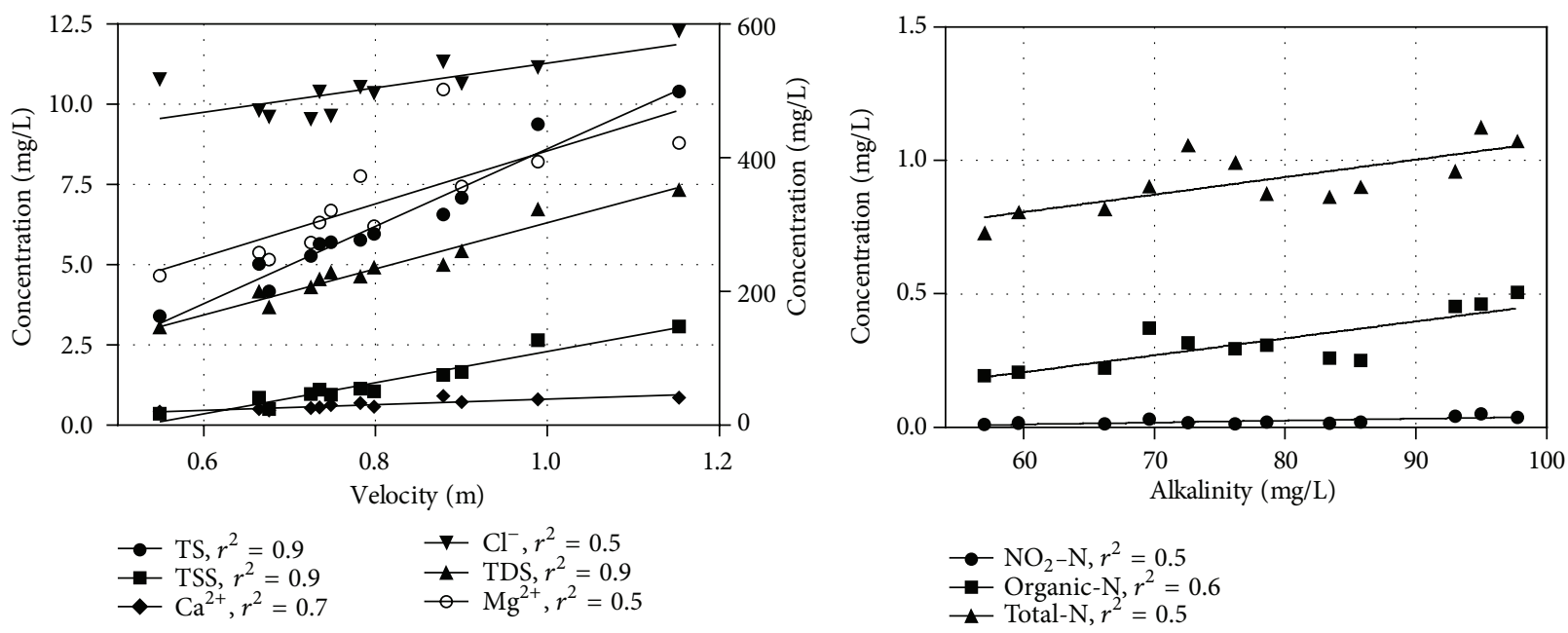

(g)

(h)
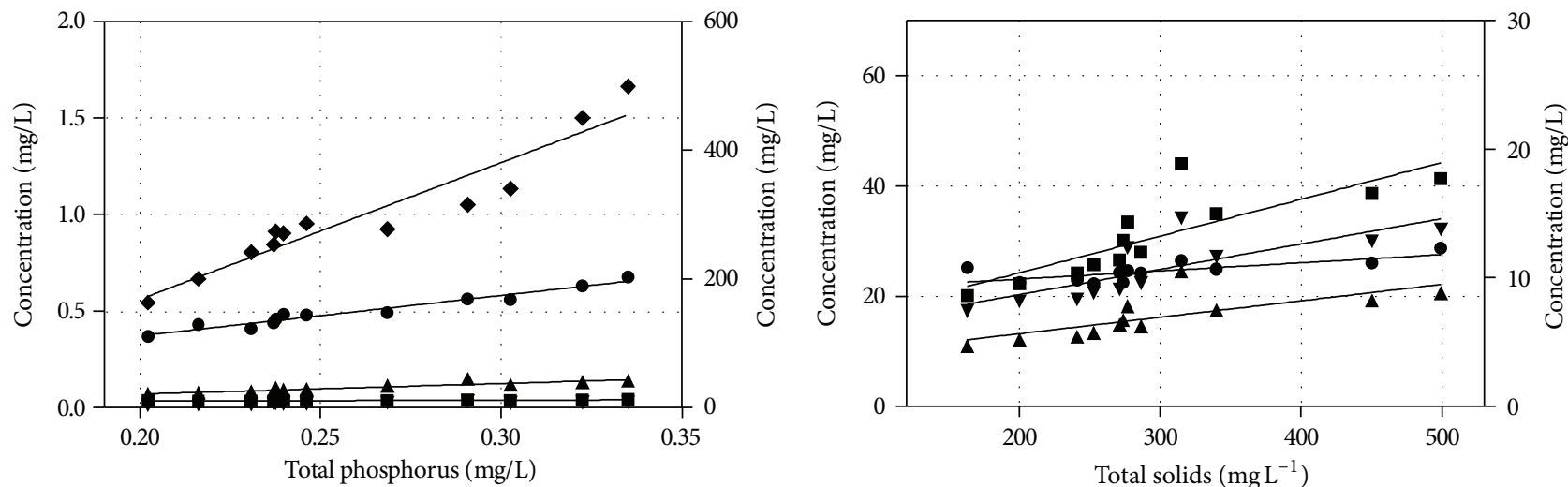

$\begin{array}{ll}\rightarrow \mathrm{NO}_{3}-\mathrm{N}, r^{2}=0.9 & \rightarrow \mathrm{TS}, r^{2}=0.9 \\ \rightarrow-\mathrm{Cl}^{2},^{2}=0.5 & \rightarrow \mathrm{Mg}^{2+}, r^{2}=0.6 \\ \leftarrow \mathrm{Ca}^{2+}, r^{2}=0.8 & \end{array}$

(i)

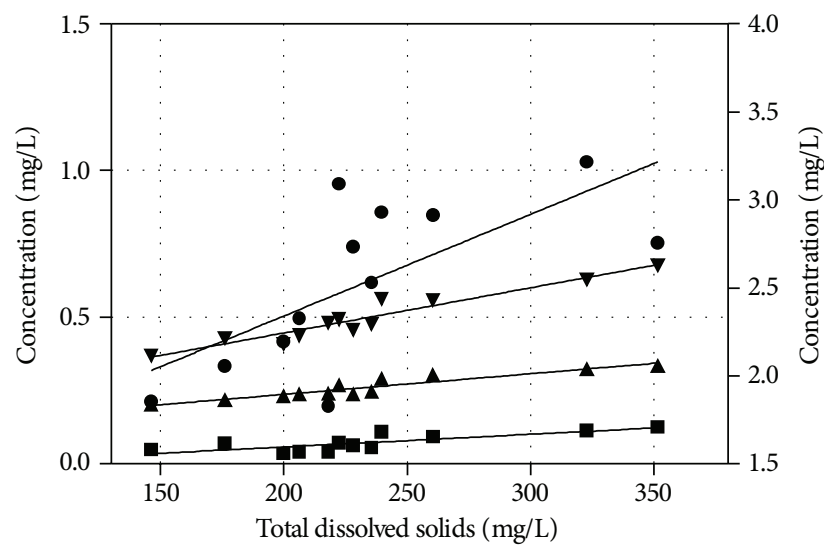

$\begin{array}{ll}\rightarrow \text { Ortho-P, } r^{2}=0.6 & \rightarrow \mathrm{NO}_{3}-\mathrm{N}, r^{2}=0.9 \\ \rightarrow \text { Total-P, } r^{2}=0.8 & \rightarrow-\mathrm{K}^{+}, r^{2}=0.4\end{array}$

(k)

$$
\begin{aligned}
& \rightarrow \mathrm{Cl}^{-}, r^{2}=0.5 \\
& \varangle \mathrm{TDS}, r^{2}=0.9 \\
& -0-\mathrm{Mg}^{2+}, r^{2}=0.5
\end{aligned}
$$

- Organic-N $r^{2}=0.6$

- Total-N, $r^{2}=0.5$

$$
\begin{array}{ll}
\longrightarrow \mathrm{Cl}^{-}, r^{2}=0.5 & \rightarrow \\
\rightarrow \mathrm{Ca}^{+}, r^{2}=0.6 \\
\rightarrow \mathrm{Mg}^{2+}, r^{2}=0.5
\end{array}
$$

(j)

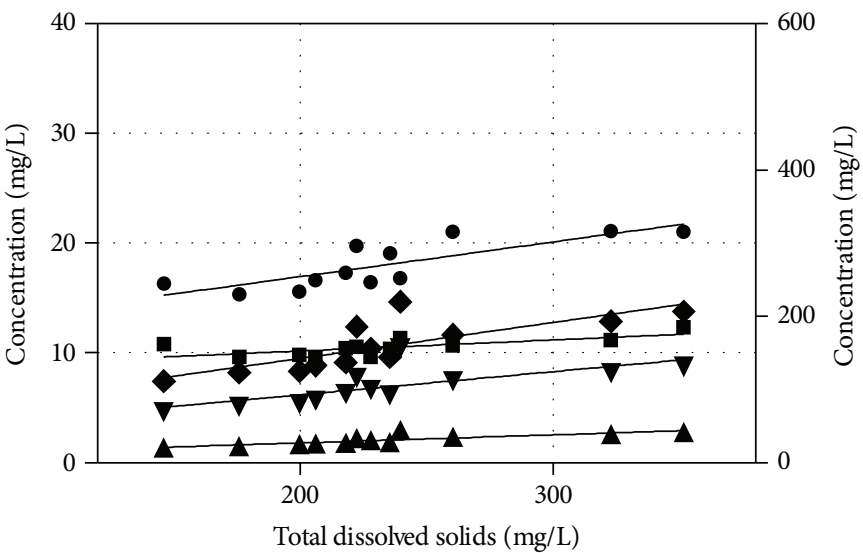

$\begin{array}{ll}\longrightarrow \text { Cond., } r^{2}=0.65 & \rightarrow \mathrm{Cl}^{-}, r^{2}=0.4 \\ \longleftarrow \mathrm{Ca}^{2+}, r^{2}=0.6 & \rightarrow \mathrm{Mg}^{2+}, r^{2}=0.5 \\ \rightarrow \mathrm{Na}^{+}, r^{2}=0.6 & \end{array}$

(l)

FIGURE 2: Plots of water quality parameters as a linear regression model. 
TABLE 3: The results of the statistical analysis with the general linear regression model to delineate the nature and magnitude of relationship among physicochemical parameters of Sukhnag stream. The regression parameters, $\alpha$ and $\beta$, were estimated from $C=\alpha+\beta \log _{e}(X)$. $R^{2}$ refers to the regression analysis.

\begin{tabular}{|c|c|c|c|c|c|c|c|c|}
\hline & Parameters & No. of $X$ values & $\alpha(\mathrm{mg} / \mathrm{L})$ & $\beta$ & $R^{2}$ & $F$ & $P$ value & Deviation from zero \\
\hline \multirow{5}{*}{ Water temperature } & $\mathrm{DO}$ & 12 & 12.05 & -0.16 & 0.8 & 53.8 & $<0.0001$ & Significant \\
\hline & Free $\mathrm{CO}_{2}$ & 12 & 7.54 & 0.06 & 0.6 & 17.9 & 0 & Significant \\
\hline & Nitrite & 12 & 0 & 0 & 0.6 & 15 & 0 & Significant \\
\hline & Ammonia & 12 & 0.14 & 0 & 0.7 & 20.1 & 0 & Significant \\
\hline & Organic-N & 12 & 0.15 & 0.01 & 0.8 & 44.3 & $<0.0001$ & Significant \\
\hline \multirow{12}{*}{ Depth } & Velocity & 12 & 0.46 & 0.2 & 0.9 & 132 & $<0.0001$ & Significant \\
\hline & OP & 12 & 0.02 & 0.03 & 0.7 & 20.6 & 0 & Significant \\
\hline & T phosphorus & 12 & 0.17 & 0.05 & 0.9 & 134 & $<0.0001$ & Significant \\
\hline & Nitrate & 12 & 0.31 & 0.11 & 0.9 & 117 & $<0.0001$ & Significant \\
\hline & Chloride & 12 & 9.23 & 0.76 & 0.5 & 11.1 & 0.01 & Significant \\
\hline & $\mathrm{Ca}$ & 12 & 16.67 & 8.34 & 0.7 & 24.8 & 0 & Significant \\
\hline & $\mathrm{Mg}$ & 12 & 4.19 & 1.61 & 0.6 & 12.1 & 0.01 & Significant \\
\hline & $\mathrm{Na}$ & 12 & 6.47 & 2.45 & 0.6 & 18.1 & 0 & Significant \\
\hline & $\mathrm{K}$ & 12 & 1.79 & 0.44 & 0.5 & 11.2 & 0.01 & Significant \\
\hline & T solids & 12 & 95.24 & 120 & 1 & 257 & $<0.0001$ & Significant \\
\hline & TSS & 12 & -18.43 & 48.6 & 1 & 215 & $<0.0001$ & Significant \\
\hline & TDS & 12 & 113.7 & 71.2 & 1 & 199 & $<0.0001$ & Significant \\
\hline \multirow{4}{*}{ Dissolved oxygen } & Free $\mathrm{CO}_{2}$ & 12 & 12.18 & -0.39 & 0.8 & 45.4 & $<0.0001$ & Significant \\
\hline & Alkalinity & 12 & 155.8 & -7.92 & 0.6 & 15.5 & 0 & Significant \\
\hline & Ammoniacal-N & 12 & -0.17 & 0.03 & 0.7 & 20.1 & 0 & Significant \\
\hline & Organic-N & 12 & 1.09 & -0.08 & 1 & 229 & $<0.0001$ & Significant \\
\hline \multirow{7}{*}{ Conductivity } & Total-P & 12 & -0.03 & 0 & 0.7 & 24.5 & 0 & Significant \\
\hline & Nitrate-N & 12 & -0.1 & 0 & 0.7 & 18.4 & 0 & Significant \\
\hline & $\mathrm{K}$ & 12 & -0.17 & 0.01 & 0.5 & 9.53 & 0.01 & Significant \\
\hline & TDS & 12 & -141.6 & 1.39 & 0.7 & 19.4 & 0 & Significant \\
\hline & Total hardness & 12 & -145.5 & 1.05 & 0.9 & 60.1 & $<0.0001$ & Significant \\
\hline & Ca hardness & 12 & -40.26 & 0.42 & 0.8 & 30.3 & 0 & Significant \\
\hline & Mg hardness & 12 & -25.57 & 0.15 & 0.8 & 38.4 & 0 & Significant \\
\hline \multirow{11}{*}{ Velocity } & $\mathrm{OP}$ & 12 & -0.06 & 0.17 & 0.8 & 31.2 & 0 & Significant \\
\hline & Total-P & 12 & 0.06 & 0.26 & 0.9 & 124 & $<0.0001$ & Significant \\
\hline & Nitrate-N & 12 & 0.05 & 0.56 & 1 & 319 & $<0.0001$ & Significant \\
\hline & $\mathrm{Na}$ & 12 & 0.6 & 12.5 & 0.7 & 24.8 & 0 & Significant \\
\hline & $\mathrm{K}$ & 12 & 0.88 & 2.07 & 0.5 & 9.61 & 0.01 & Significant \\
\hline & Total solids & 12 & -165.3 & 579 & 1 & 211 & $<0.0001$ & Significant \\
\hline & TSS & 12 & -123 & 233 & 0.9 & 147 & $<0.0001$ & Significant \\
\hline & TDS & 12 & -462.28 & 345 & 1 & 199 & $<0.0001$ & Significant \\
\hline & Chloride & 12 & 7.46 & 3.82 & 0.6 & 13.1 & 0 & Significant \\
\hline & $\mathrm{Ca}$ & 12 & -2.7 & 41.8 & 0.8 & 32.2 & 0 & Significant \\
\hline & $\mathrm{Mg}$ & 12 & 0.3 & 8.25 & 0.6 & 15.9 & 0 & Significant \\
\hline \multirow{3}{*}{ Alkalinity } & Nitrate-N & 12 & -0.03 & 0 & 0.6 & 12 & 0.01 & Significant \\
\hline & Organic-N & 12 & 0.17 & 0.01 & 0.7 & 18.9 & 0 & Significant \\
\hline & Total-N & 12 & 0.41 & 0.01 & 0.6 & 12.3 & 0.01 & Significant \\
\hline \multirow{5}{*}{$\mathrm{T}$ phosphorus } & Nitrate-N & 12 & -0.05 & 2.09 & 1 & 221 & $<0.0001$ & Significant \\
\hline & Chloride & 12 & 6.67 & 14.7 & 0.6 & 14.5 & 0 & Significant \\
\hline & $\mathrm{Ca}$ & 12 & -12.49 & 166 & 0.8 & 53.8 & $<0.0001$ & Significant \\
\hline & $\mathrm{Mg}$ & 12 & -1.76 & 33.2 & 0.7 & 23.3 & 0 & Significant \\
\hline & Total solids & 12 & -256.5 & 2125 & 0.9 & 94.7 & $<0.0001$ & Significant \\
\hline
\end{tabular}


TABLe 3: Continued.

\begin{tabular}{|c|c|c|c|c|c|c|c|c|}
\hline & Parameters & No. of $X$ values & $\alpha(\mathrm{mg} / \mathrm{L})$ & $\beta$ & $R^{2}$ & $F$ & $P$ value & Deviation from zero \\
\hline \multirow{4}{*}{ T solids } & Chloride & 12 & 8.63 & 0.01 & 0.6 & 12 & 0.01 & Significant \\
\hline & $\mathrm{Ca}$ & 12 & 10.86 & 0.07 & 0.7 & 21.6 & 0 & Significant \\
\hline & $\mathrm{Mg}$ & 12 & 3.09 & 0.01 & 0.5 & 10.8 & 0.01 & Significant \\
\hline & $\mathrm{Na}$ & 12 & 4.74 & 0.02 & 0.6 & 16.4 & 0 & Significant \\
\hline \multirow{9}{*}{ TDS } & $\mathrm{K}$ & 12 & 1.19 & 0.01 & 0.5 & 9.1 & 0.01 & Significant \\
\hline & Ortho-P & 12 & -0.03 & 0 & 0.6 & 18.1 & 0 & Significant \\
\hline & Total-P & 12 & 0.1 & 0 & 0.9 & 76.7 & $<0.0001$ & Significant \\
\hline & Nitrate-N & 12 & 0.14 & 0 & 0.9 & 117 & $<0.0001$ & Significant \\
\hline & Conductivity & 12 & 159.2 & 0.48 & 0.7 & 19.4 & 0 & Significant \\
\hline & Chloride & 12 & 8.16 & 0.01 & 0.5 & 9.67 & 0.01 & Significant \\
\hline & $\mathrm{Ca}$ & 12 & 4.89 & 0.11 & 0.7 & 20.1 & 0 & Significant \\
\hline & $\mathrm{Mg}$ & 12 & 1.97 & 0.02 & 0.5 & 10.1 & 0.01 & Significant \\
\hline & $\mathrm{Na}$ & 12 & 2.98 & 0.03 & 0.6 & 15.4 & 0 & Significant \\
\hline
\end{tabular}

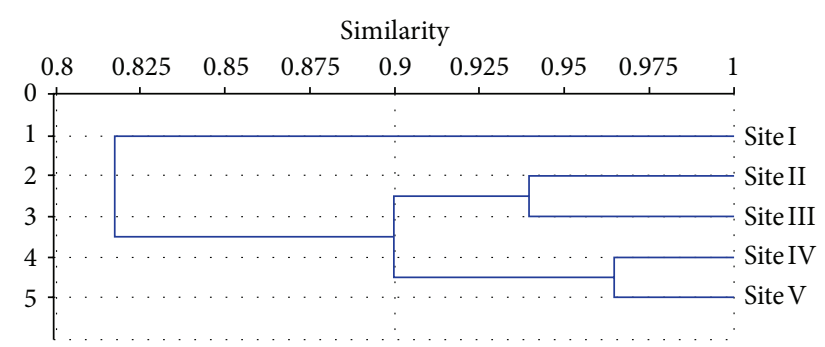

Figure 3: Bray-Curtis cluster analyses of five study sites.

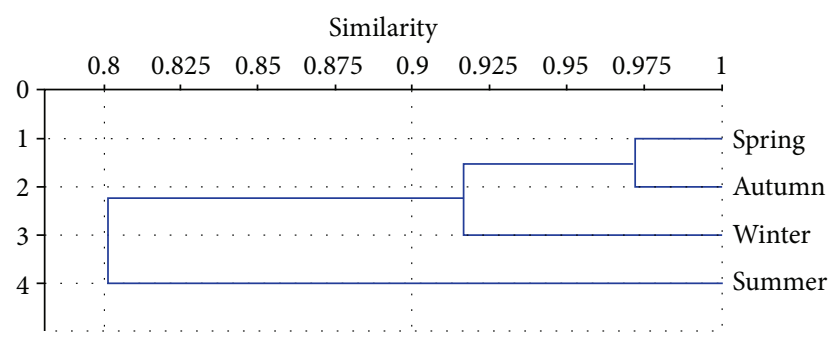

FIGURE 4: Bray-Curtis cluster analyses of four study seasons.

component. It also reflects that overloadings of total- $\mathrm{P}, \mathrm{NO}_{3}-$ $\mathrm{N}, \mathrm{Ca}^{2+}, \mathrm{Na}^{+}$, TS, TSS, and TDS are responsible for the heaviest pollution problem in the stream. For factor $2, \mathrm{NO}_{2}-$ $\mathrm{N}$, free- $\mathrm{CO}_{2}$, and organic- $\mathrm{N}$ have the highest factor loading value ( $>0.96$ ), suggesting that organic-N pollution is also a major environmental pollutant in rivers. Factor loadings can be interpreted as the correlation between the factors and the variables, that is, physicochemical parameters.

To determine which sampling variables were closely related, a plot of factor coordinates for all significant observations (cases) was constructed using the factors obtained from factor loading analysis (Figure 6). Figure 7 shows the cluster of seasons which gets affected by all 26 physicochemical parameters. The variable lines were obtained from the factor loadings of the original variables. They stand for the contribution of the variables to the samples. The closer the two

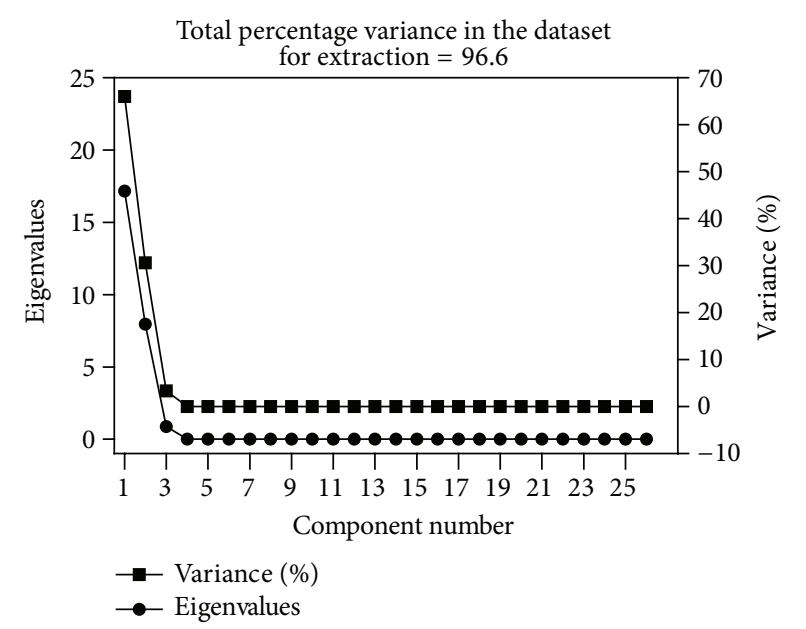

FIgURE 5: Score plot of eigenvalues versus components along with $\%$ variance components in Sukhnag stream.

variable lines, the stronger the mutual correlation [47]. The sampling sites (cases) that are clustered near each other have similar characteristics with respect to the factors. The positive loading of temperature is associated with seasonal variation [48]. The inverse relationship between temperature and dissolved oxygen is a natural process in water [49], because warm water easily becomes saturated with oxygen and thus holds less DO [50]. At higher temperature, the solubility of calcium decreases [51]. Positive loading of $\mathrm{NO}_{3}-\mathrm{N}$, total-N, alkalinity, free $\mathrm{CO}_{2}, \mathrm{Ca}^{2+}$, and $\mathrm{Na}^{+}$indicates organic matter from domestic wastewaters [52]. Positive loading of $\mathrm{Ca}^{2+}$ and $\mathrm{Na}^{+}$is attributed to agricultural runoff [53], while as asserted seasonal variations of $\mathrm{Ca}, \mathrm{Mg}$, and $\mathrm{K}$ are linked with parent rock materials in the catchment area [54]. Positive loading of $\mathrm{NO}_{3}-\mathrm{N}$ and total-P in spring and summer season has been also associated with agricultural runoff [55]. During these seasons, farmers use reckless fertilizers and pesticides, which represent point and nonpoint source pollution from orchard and agriculture areas. Negative loading of $\mathrm{pH}$ and $\mathrm{DO}(-0.9)$ 


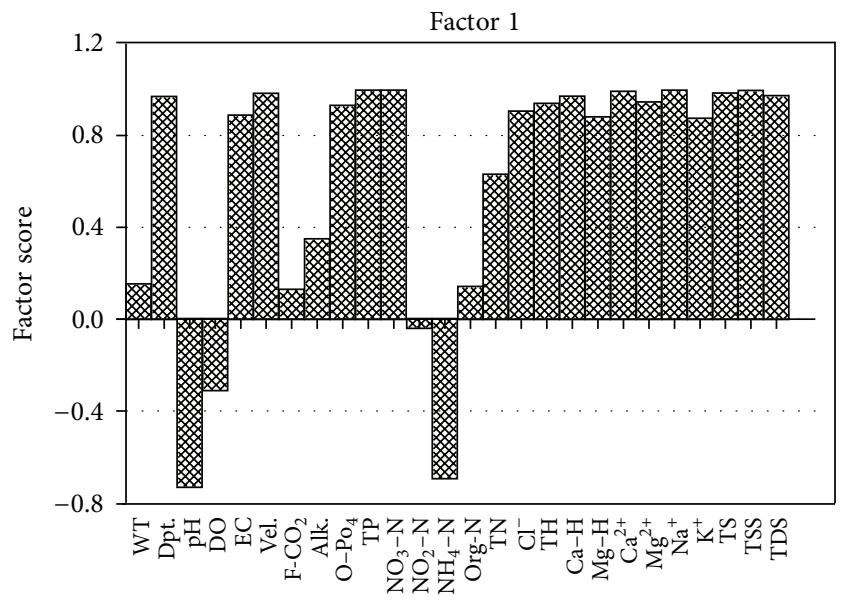

(a)

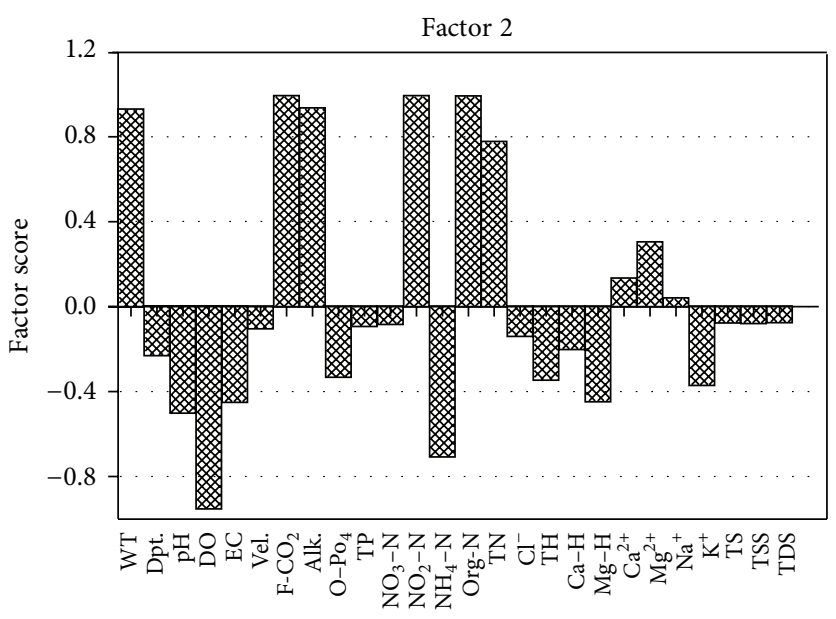

(b)

FIGURE 6: Factor score for factors 1 and 2 in the Sukhnag stream.

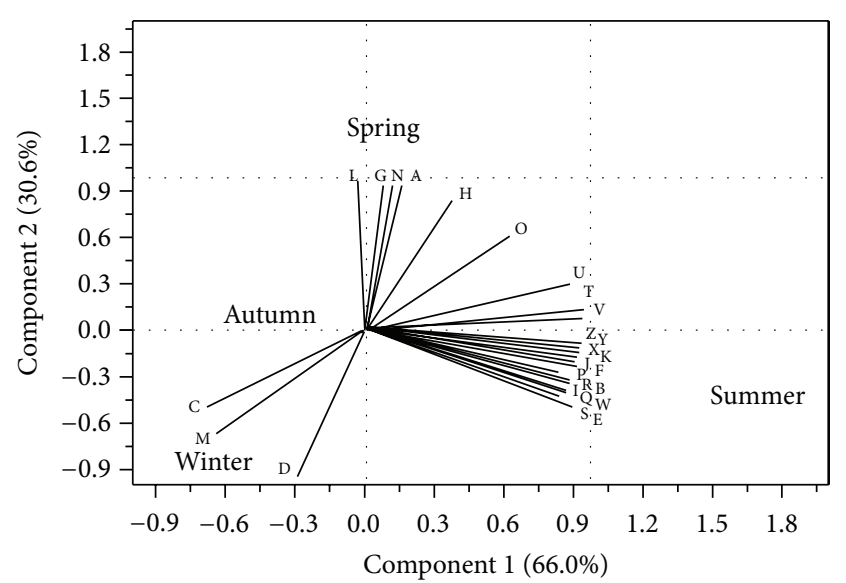

FIGURE 7: Bi plots for principal component analysis $1+2$ of water quality parameters (where $\mathrm{A}=$ water temperature $\left({ }^{\circ} \mathrm{C}\right), \mathrm{B}=$ depth $(\mathrm{m}), \mathrm{C}=\mathrm{pH}, \mathrm{D}=$ dissolved oxygen $\left(\mathrm{mg} \mathrm{L}^{-1}\right), \mathrm{E}=$ electrical conductivity, $\mathrm{F}=$ velocity $\left(\mathrm{mg} \mathrm{L}^{-1}\right), \mathrm{G}=$ free $\mathrm{CO}_{2}\left(\mathrm{mg} \mathrm{L}^{-1}\right), \mathrm{H}$ = alkalinity $\left(\mathrm{mg} \mathrm{L}^{-1}\right), \mathrm{I}=$ orthophosphorus $\left(\mathrm{mg} \mathrm{L}^{-1}\right), \mathrm{J}=$ total phosphorus $\left(\mathrm{mg} \mathrm{L}^{-1}\right), \mathrm{K}=\mathrm{NO}_{3}-\mathrm{N}\left(\mathrm{mg} \mathrm{L}^{-1}\right), \mathrm{L}=\mathrm{NO}_{2}-\mathrm{N}\left(\mathrm{mg} \mathrm{L}^{-1}\right)$, $\mathrm{M}=\mathrm{NH}_{4}-\mathrm{N}\left(\mathrm{mg} \mathrm{L}^{-1}\right), \mathrm{N}=$ organic nitrogen $\left(\mathrm{mg} \mathrm{L}^{-1}\right), \mathrm{O}=$ total nitrogen $\left(\mathrm{mg} \mathrm{L}^{-1}\right), \mathrm{P}=\mathrm{Cl}^{-}\left(\mathrm{mg} \mathrm{L}^{-1}\right), \mathrm{Q}=$ total hardness $\left(\mathrm{mg} \mathrm{L}^{-1}\right), \mathrm{R}=$ Ca hardness $\left(\mathrm{mg} \mathrm{L}^{-1}\right), \mathrm{S}=\mathrm{Mg}$ hardness $\left(\mathrm{mg} \mathrm{L}^{-1}\right), \mathrm{T}=\mathrm{Ca}^{2+}\left(\mathrm{mg} \mathrm{L}^{-1}\right)$, $\mathrm{U}=\mathrm{Mg}^{2+}\left(\mathrm{mg} \mathrm{L}^{-1}\right), \mathrm{V}=\mathrm{Na}^{+}\left(\mathrm{mg} \mathrm{L}^{-1}\right), \mathrm{W}=\mathrm{K}^{+}\left(\mathrm{mg} \mathrm{L}^{-1}\right), \mathrm{X}=$ total solids $\left(\mathrm{mg} \mathrm{L}^{-1}\right), \mathrm{Y}=$ total suspended solids $\left(\mathrm{mg} \mathrm{L}^{-1}\right)$, and $\mathrm{Z}=$ total dissolved solids $\left(\mathrm{mg} \mathrm{L}^{-1}\right)$.

in factor 1 with positive scores of TN, TP, and inorganic nutrients represented anthropogenic pollution sources as high levels of dissolved organic matter consume large amounts of oxygen for decomposition leading to formation of organic acids, $\mathrm{CO}_{2}$, and ammonia. Hydrolysis of these acids, dissolution of $\mathrm{CO}_{2}$ in water column, and/or oxidation of $\mathrm{NH}_{4}$ ions under oxic conditions by the nitrification processes created a decrease in water $\mathrm{pH}$ [56-58]. From the physicochemical data matrix, it was found that the TSS load was highest at
TABLE 4: Loadings of 26 experimental variables on principal components for Sukhnag stream.

\begin{tabular}{|c|c|c|}
\hline Variables & Factor 1 & Factor 2 \\
\hline WT & 0.1513 & 0.9283 \\
\hline Depth & 0.9684 & -0.2298 \\
\hline $\mathrm{pH}$ & -0.9312 & -0.4996 \\
\hline DO & -0.9309 & -0.7505 \\
\hline $\mathrm{EC}$ & 0.8865 & -0.449 \\
\hline Velocity & 0.9814 & -0.1033 \\
\hline $\mathrm{F}-\mathrm{CO}_{2}$ & 0.1286 & 0.9913 \\
\hline Alkalinity & 0.3485 & 0.9345 \\
\hline $\mathrm{O}-\mathrm{PO}_{4}$ & 0.9291 & -0.3316 \\
\hline $\mathrm{TP}$ & 0.9957 & -0.09201 \\
\hline $\mathrm{NO}_{3}-\mathrm{N}$ & 0.9961 & -0.08224 \\
\hline $\mathrm{NO}_{2}-\mathrm{N}$ & -0.03837 & 0.9913 \\
\hline $\mathrm{NH}_{4}-\mathrm{N}$ & -0.6931 & -0.7058 \\
\hline Org-N & 0.1408 & 0.9899 \\
\hline $\mathrm{TN}$ & 0.6293 & 0.7754 \\
\hline $\mathrm{Cl}^{-}$ & 0.9045 & -0.1383 \\
\hline $\mathrm{TH}$ & 0.9382 & -0.3449 \\
\hline $\mathrm{Ca}-\mathrm{H}$ & 0.9689 & -0.2011 \\
\hline $\mathrm{Mg}-\mathrm{H}$ & 0.8792 & -0.4466 \\
\hline $\mathrm{Ca}^{2+}$ & 0.9912 & 0.1325 \\
\hline $\mathrm{Mg}^{2+}$ & 0.9444 & 0.3026 \\
\hline $\mathrm{Na}^{+}$ & 0.9956 & 0.03841 \\
\hline $\mathrm{K}^{+}$ & 0.8731 & -0.3702 \\
\hline TS & 0.9837 & -0.07564 \\
\hline TSS & 0.9944 & -0.07786 \\
\hline TDS & 0.9715 & -0.07375 \\
\hline
\end{tabular}

downward sampling sites due to possible contribution from nonpoint sources, most probably from land drainage [59]. 


\section{Conclusion}

This research assessed the linkages between spatio-temporal variability and water quality using multivariate statistics in the Sukhnag stream, Kashmir Himalayas. All sampled parameters indicated significant spatiotemporal variability. From multivariate analysis it could be construed that the stream water quality is primarily influenced by agricultural runoff and wastewater discharge. The results from PCA suggested that most of the variations in water quality are explained by the natural soluble salts, nonpoint source nutrients, and anthropogenic organic pollutants. Results of regression analysis clearly showed that, in peak flow season, runoff raises the concentration of most of the inorganic and organic parameters. The increasing level of pollution from the head of the stream to the tail indicated progressive anthropogenic pressure in the downstream areas. The outcomes of the current research shall help in providing a holistic watershed management plan to recover the depreciating water quality of this important stream, which directly drains into the Lake Wular which is a Ramsar site. In order to halt and reverse the deteriorating water quality of this stream, we recommend stoppage of crop cultivation along the slopes (particularly, maize), regulating the use of excessive fertilizers in the agriculture, and setting up of sewage treatment system in the residential areas.

\section{Conflict of Interests}

The authors declare that there is no conflict of interests regarding the publication of this paper.

\section{Acknowledgments}

This work is part of the Ph.D. research of Salim Aijaz Bhat for which he is indebted to Dr. Azra N. Kamili, Professor and Director, Centre of Research for Development (CORD), and Head, Department of Environmental Science, University of Kashmir, for providing full support and necessary laboratory facilities for carrying out the chemical analysis. The authors would like to acknowledge the support of Dr. Shakil A. Romshoo, Professor and Head, Department of Earth Sciences, University of Kashmir, for providing facilities for the generation of maps and figures. Also the authors acknowledge the help from the State Irrigation \& Flood Control and Meteorological Department for providing necessary data.

\section{References}

[1] H. P. Jarvie, B. A. Whitton, and C. Neal, "Nitrogen and phosphorus in east coast British rivers: speciation, sources and biological significance," Science of the Total Environment, vol. 210211, pp. 79-109, 1998.

[2] V. Simeonov, P. Simeonova, and R. Tsitouridou, "Chemometric quality assessment of surface waters two case studies," Chemical and Engineering Ecology, vol. 11, no. 6, pp. 449-469, 2004.

[3] A. H. Mahvi, J. Nouri, A. A. Babaei, and R. Nabizadeh, "Agricultural activities impact on groundwater nitrate pollution," International Journal of Environmental Science and Technology, vol. 2, no. 1, pp. 41-47, 2005.
[4] J. Nouri, A. R. Karbassi, and S. Mirkia, "Environmental management of coastal regions in the Caspian Sea," International Journal of Environmental Science and Technology, vol. 5, no. 1, pp. 43-52, 2008.

[5] A. R. Karbassi, S. M. Monavari, G. R. Nabi Bidhendi, J. Nouri, and K. Nematpour, "Metal pollution assessment of sediment and water in the Shur River," Environmental Monitoring and Assessment, vol. 147, no. 1-3, pp. 107-116, 2008.

[6] S. S. Vutukuru, "Chromium induced alterations in some biochemical profiles of the Indian major carp, Labeo rohita (Hamilton)," Bulletin of Environmental Contamination and Toxicology, vol. 70, no. 1, pp. 118-123, 2003.

[7] J. C. Vie, C. Hilton-Taylor, and S. N. Stuart, Eds., Wildlife in a Changing World: An Analysis of the 2008 IUCN Red List of ThraAtened Species, IUCN, Gland, Switzerland, 2009.

[8] A. O. Adeogun, A. V. Chukwuka, and O. R. Ibor, "Impact of abbatoir and saw mill effluents on water quality of Upper Ogun River (Abeokuta)," The American Journal of Environmental Sciences, vol. 7, no. 6, pp. 525-530, 2011.

[9] S. Monavari and B. Guieysse, "Development of water quality test kit based on substrate utilization and toxicity resistance in river microbial communities," International Journal of Environmental Research, vol. 1, no. 2, pp. 136-142, 2007.

[10] R. B. Khadka and A. B. Khanal, "Environmental management plan (EMP) for melamchi water supply project, Nepal," International Journal of Environmental Research, vol. 2, no. 1, pp. 87-96, 2008.

[11] F. Altaf, G. Meraj, and S. A. Romshoo, "Morphometric Analysis to Infer Hydrological Behaviour of Lidder Watershed, Western Himalaya, India," Geography Journal, vol. 2013, Article ID 178021, 2013.

[12] A. H. Mtethiwa, A. Munyenyembe, W. Jere, and E. Nyali, "Efficiency of oxidation ponds in wastewater treatment," International Journal of Environmental Research, vol. 2, no. 2, pp. 149$152,2008$.

[13] J. R. Chinhanga, "Impact of industrial effluent from an iron and steel company on the physicochemical quality of Kwekwe River water in Zimbabwe," International Journal of Engineering, Science and Technology, vol. 2, no. 7, pp. 29-40, 2010.

[14] USEPA, Recent Recommended Water Quality Criteria, United States Environmental Protection Agency, 2007.

[15] S. Wei, Y. Wang, J. C. W. Lam et al., "Historical trends of organic pollutants in sediment cores from Hong Kong," Marine Pollution Bulletin, vol. 57, no. 6-12, pp. 758-766, 2008.

[16] K. N. Don-Pedro, E. O. Oyewo, and A. A. Otitoloju, "Trend of heavy metal concentration in Lagos Lagoon ecosystem, Nigeria," West African Journal of Applied Ecology, vol. 5, pp. 103$114,2004$.

[17] W. Dixon and B. Chiswell, "Review of aquatic monitoring program design," Water Research, vol. 30, no. 9, pp. 1935-1948, 1996.

[18] V. Simeonov, J. A. Stratis, C. Samara et al., "Assessment of the surface water quality in Northern Greece," Water Research, vol. 37, no. 17, pp. 4119-4124, 2003.

[19] K. P. Singh, A. Malik, D. Mohan, and S. Sinha, "Multivariate statistical techniques for the evaluation of spatial and temporal variations in water quality of Gomti River (India): a case study," Water Research, vol. 38, no. 18, pp. 3980-3992, 2004.

[20] B. Helena, R. Pardo, M. Vega, E. Barrado, J. M. Fernandez, and L. Fernandez, "Temporal evolution of groundwater composition in an alluvial aquifer (Pisuerga River, Spain) by principal com- 
ponent analysis," Water Research, vol. 34, no. 3, pp. 807-816, 2000.

[21] K. P. Singh, A. Malik, V. K. Singh, N. Basant, and S. Sinha, "Multi-way modeling of hydro-chemical data of an alluvial river system-A case study," Analytica Chimica Acta, vol. 571, no. 2, pp. 248-259, 2006.

[22] S. A. Bhat, G. Meraj, S. Yaseen, A. R. Bhat, and A. K. Pandit, "Assessing the impact of anthropogenic activities on spatiotemporal variation of water quality in Anchar lake, Kashmir Himalayas," International Journal of Environmental Sciences, vol. 3, no. 5, pp. 1625-1640, 2013.

[23] F. J. H. Mackereth, Some Methods for Water Analysis for Limnologists, Freshwater Biological Association, London, UK, 1963.

[24] H. L. Golterman and R. S. Clymo, "Methods for Physical and Chemical Analysis of Freshwaters," IBP Hand Book no. 8, Blackwell Scientific Publication, Oxford, UK, 1969.

[25] American Public Health Association (A.P.H.A.), Standard Methods For Examination of Water and Waste Water, American Public Health Association, Washington, DC, USA, 20th edition, 1998.

[26] S. O. Fakayode, "Impact assessment of industrial effluent on water quality of the receiving Alaro River in Ibadan, Nigeria," African Journal of Environmental Assessment and Management, vol. 10, pp. 1-13, 2005.

[27] L. S. Ernestova and I. V. Semenova, "The self-purification capacity of natural water as an indicator of the ecological state of a water body," Water Resources, vol. 21, no. 2, pp. 146-150, 1994.

[28] J. H. Zar, Biostastical Analysis, Printice Hall, Englewood Cliffs, NJ, USA, 5th edition, 2009.

[29] Y.-H. Yang, F. Zhou, H.-C. Guo et al., "Analysis of spatial and temporal water pollution patterns in Lake Dianchi using multivariate statistical methods," Environmental Monitoring and Assessment, vol. 170, no. 1-4, pp. 407-416, 2010.

[30] G. Ragno, M. D. Luca, and G. Ioele, "An application of cluster analysis and multivariate classification methods to spring water monitoring data," Microchemical Journal, vol. 87, no. 2, pp. 119127, 2007.

[31] S. Shrestha and F. Kazama, "Assessment of surface water quality using multivariate statistical techniques: a case study of the Fuji river basin, Japan," Environmental Modelling and Software, vol. 22, no. 4, pp. 464-475, 2007.

[32] D. A. Wunderlin, D. María del Pilar, A. María Valeria, P. S. Fabiana, H. A. Cecilia, and B. María De Los Ángeles, "Pattern recognition techniques for the evaluation of spatial and temporal variations in water quality. a case study," Water Research, vol. 35, no. 12, pp. 2881-2894, 2001.

[33] M.-L. Wu and Y.-S. Wang, "Using chemometrics to evaluate anthropogenic effects in Daya Bay, China," Estuarine, Coastal and Shelf Science, vol. 72, no. 4, pp. 732-742, 2007.

[34] S. Yu, J. Shang, J. Zhao, and H. Guo, "Factor analysis ang dynamics of water quality of the Songhua river, Northeast China," Water, Air, and Soil Pollution, vol. 144, no. 1-4, pp. 159$169,2003$.

[35] C. Filik Iscen, Ö. Emiroglu, S. Ilhan, N. Arslan, V. Yilmaz, and S. Ahiska, "Application of multivariate statistical techniques in the assessment of surface water quality in Uluabat Lake, Turkey," Environmental Monitoring and Assessment, vol. 144, no. 1-3, pp. 269-276, 2008.

[36] M.-L. Wu, Y.-S. Wang, C.-C. Sun, H. Wang, Z.-P. Lou, and J.-D. Dong, "Using chemometrics to identify water quality in Daya Bay, China," Oceanologia, vol. 51, no. 2, pp. 217-232, 2009.
[37] D. Mulkerrins, A. D. W. Dobson, and E. Colleran, "Parameters affecting biological phosphate removal from wastewaters," Environment International, vol. 30, no. 2, pp. 249-259, 2004.

[38] N. M. Mattikalli and K. S. Richards, "Estimation of surface water quality changes in response to land use change: application of the export coefficient model using remote sensing and geographical information system," Journal of Environmental Management, vol. 48, no. 3, pp. 263-282, 1996.

[39] P. Basnyat, L. D. Teeter, B. G. Lockaby, and K. M. Flynn, “The use of remote sensing and GIS in watershed level analyses of non-point source pollution problems," Forest Ecology and Management, vol. 128, no. 1-2, pp. 65-73, 2000.

[40] M. J. Brogueira and G. Cabeçadas, "Identification of similar environmental areas in Tagus estuary by using multivariate analysis," Ecological Indicators, vol. 6, no. 3, pp. 508-515, 2006.

[41] A. Ogino, H. Koshikawa, T. Nakahara, and H. Uchiyama, "Succession of microbial communities during a biostimulation process as evaluated by DGGE and clone library analyses," Journal of Applied Microbiology, vol. 91, no. 4, pp. 625-635, 2001.

[42] K. Van Der Gucht, K. Sabbe, L. De Meester et al., "Contrasting bacterioplankton community composition and seasonal dynamics in two neighbouring hypertrophic freshwater lakes," Environmental Microbiology, vol. 3, no. 11, pp. 680-690, 2001.

[43] C.-H. Yang, D. E. Crowley, and J. A. Menge, "16S rDNA fingerprinting of rhizosphere bacterial communities associated with healthy and Phytophthora infected avocado roots," FEMS Microbiology Ecology, vol. 35, no. 2, pp. 129-136, 2001.

[44] R. B. Cattell and J. A. Jaspers, "A general plasmode (No. 3010-5-2) for factor analytic exercises and research," Multivariate Behavioral Research Monographs, vol. 67, p. 211, 1967.

[45] Y. Z. Liang and R. Q. Yu, Chemometrics, Central South University Press, Changsha, China, 2000.

[46] R. Aruga, G. Negro, and G. Ostacoli, "Multivariate data analysis applied to the investigation of river pollution," Fresenius' Journal of Analytical Chemistry, vol. 346, no. 10-11, pp. 968-975, 1993.

[47] W. Qu and P. Kelderman, "Heavy metal contents in the Delft canal sediments and suspended solids of the River Rhine: multivariate analysis for source tracing," Chemosphere, vol. 45, no. 67, pp. 919-925, 2001.

[48] P. R. Jayaraman, T. Ganga Devi, and T. Vasudevan Nayar, "Water quality studies on Karamana river, Thiruvananthapuram district, South Kerala, India," Pollution Research, vol. 22, no. 1, pp. 89-100, 2003.

[49] V. R. Solanki, M. M. Hussain, and S. S. Raja, "Water quality assessment of Lake Pandu Bodhan, Andhra Pradesh State, India," Environmental Monitoring and Assessment, vol. 163, no. 1-4, pp. 411-419, 2010.

[50] M.-L. Wu, Y.-S. Wang, C.-C. Sun, H. Wang, J.-D. Dong, and S.H. Han, "Identification of anthropogenic effects and seasonality on water quality in Daya Bay, South China Sea," Journal of Environmental Management, vol. 90, no. 10, pp. 3082-3090, 2009.

[51] M. K. Jyoti and R. Akhtar, "Some limnological investigations of Sarkoot pond located in Kishtwar district Doda, J and K State," Journal of Research and Development, vol. 7, pp. 27-34, 2007.

[52] S. Shrestha and F. Kazama, "Assessment of surface water quality using multivariate statistical techniques: a case study of the Fuji river basin, Japan," Environmental Modelling and Software, vol. 22, no. 4, pp. 464-475, 2007. 
[53] H. Juahir, S. M. Zain, M. K. Yusoff et al., "Spatial water quality assessment of Langat River Basin (Malaysia) using environmetric techniques," Environmental Monitoring and Assessment, vol. 173, no. 1-4, pp. 625-641, 2011.

[54] M. A. Khan, M. A. Shah, S. S. Mir, and S. Bashir, "The environmental status of a Kashmir Himalayan wetland game reserve: aquatic plant communities and eco-restoration measures," Lakes and Reservoirs, vol. 9, no. 2, pp. 125-132, 2004.

[55] D. G. Uzarski, T. M. Burton, M. J. Cooper, J. W. Ingram, and S. T. A. Timmermans, "Fish habitat use within and across wetland classes in coastal wetlands of the five great lakes: development of a Fish-based index of biotic integrity," Journal of Great Lakes Research, vol. 31, no. 1, pp. 171-187, 2005.

[56] M. Vega, R. Pardo, E. Barrado, and L. Debán, “Assessment of seasonal and polluting effects on the quality of river water by exploratory data analysis," Water Research, vol. 32, no. 12, pp. 3581-3592, 1998.

[57] R.-H. Kim, J. Lee, and H.-W. Chang, "Characteristics of organic matter as indicators of pollution from small-scale livestock and nitrate contamination of shallow groundwater in an agricultural area," Hydrological Processes, vol. 17, no. 12, pp. 2485-2496, 2003.

[58] J.-H. Kim, R.-H. Kim, J. Lee, T.-J. Cheong, B.-W. Yum, and H.W. Chang, "Multivariate statistical analysis to identify the major factors governing groundwater quality in the coastal area of Kimje, South Korea," Hydrological Processes, vol. 19, no. 6, pp. 1261-1276, 2005.

[59] S. K. Sundaray, U. C. Panda, B. B. Nayak, and D. Bhatta, "Behaviour and distribution pattern of nutrients in river-estua-rine waters of Mahanadi, Orissa, India," Asian Journal of Water, Environment and Pollution, vol. 2, no. 1, pp. 77-84, 2005. 

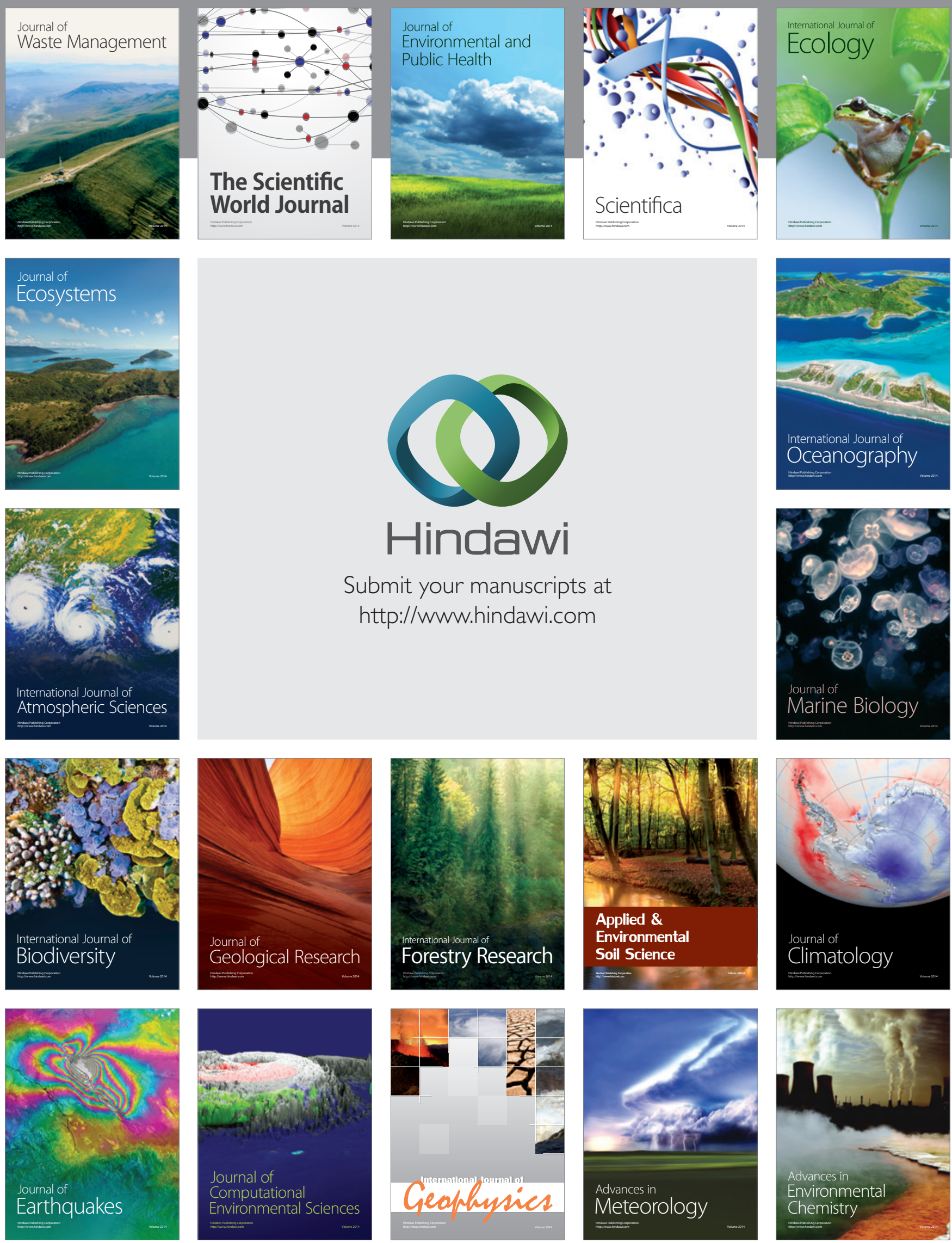